OPEN ACCESS

Edited by:

Lei Xue,

Tongji University, China

Reviewed by:

Ranhui Duan,

Central South University, China Shupeng Li,

Peking University, China

*Correspondence:

Gong Ping Liu

liugp111@mail.hust.edu.cn

Gang Li

gangli2008@hotmail.com

tThese authors have contributed equally to this work and share first authorship

Specialty section:

This article was submitted to Molecular and Cellular Pathology,

a section of the journal

Frontiers in Cell and Developmental

Biology

Received: 05 July 2021 Accepted: 25 November 2021

Published: 04 January 2022

Citation:

Jiang $X J, W u Y Q, M a R$, Chang $Y M$, Li LL, Zhu JH, Liu GP and Li G (2022)

PINK1 Alleviates Cognitive

Impairments via Attenuating

Pathological Tau Aggregation in a

Mouse Model of Tauopathy.

Front. Cell Dev. Biol. 9:736267.

doi: 10.3389/fcell.2021.736267

\section{PINK1 Alleviates Cognitive Impairments via Attenuating Pathological Tau Aggregation in a Mouse Model of Tauopathy}

\author{
Xing Jun Jiang ${ }^{1 \dagger}$, Yan Qing $W u^{1 \dagger}$, Rong $\mathrm{Ma}^{2}$, Yan Min Chang ${ }^{1}$, Lu Lu $\mathrm{Li}^{1}$, Jia Hui Zhu ${ }^{1}$, \\ Gong Ping Liu ${ }^{3,4 *}$ and Gang $L i^{1 *}$
}

${ }^{1}$ Department of Neurology, Union Hospital, Tongji Medical College, Huazhong University of Science and Technology, Wuhan, China, ${ }^{2}$ Department of Pharmacology, School of Basic Medicine, Tongji Medical College, Huazhong University of Science and Technology, Wuhan, China, ${ }^{3}$ Department of Pathophysiology, School of Basic Medicine and the Collaborative Innovation Center for Brain Science, Key Laboratory of Ministry of Education of China and Hubei Province for Neurological Disorders, Tongji Medical College, Huazhong University of Science and Technology, Wuhan, China, ${ }^{4}$ Co-Innovation Center of Neuroregeneration, Nantong University, Nantong, China

As a primary cause of dementia and death in older people, Alzheimer's disease (AD) has become a common problem and challenge worldwide. Abnormal accumulation of tau proteins in the brain is a hallmark pathology of $A D$ and is closely related to the clinical progression and severity of cognitive deficits. Here, we found that overexpression of phosphatase and tensin homolog (PTEN)-induced kinase 1 (PINK1) effectively promoted the degradation of tau, thereby rescuing neuron loss, synaptic damage, and cognitive impairments in a mouse model of tauopathy with AAV-full-length human Tau ( $\mathrm{hTau}$ ) injected into the hippocampal CA1 area (hTau mice). Overexpression of PINK1 activated autophagy, and chloroquine but not MG132 reversed the PINK1-induced decrease in human Tau levels and cognitive improvement in hTau mice. Furthermore, PINK1 also ameliorated mitochondrial dysfunction induced by hTau. Taken together, our data revealed that PINK1 overexpression promoted degradation of abnormal accumulated tau via the autophagy-lysosome pathway, indicating that PINK1 may be a potential target for AD treatment.

\section{Keywords: tau, PINK1, autophagy, memory, Alzheimer's disease}

\section{INTRODUCTION}

Tauopathies are a group of human neurological disorders, which are pathologically characterized by abnormal accumulation of tau filaments in the brain. Among the tauopathies, Alzheimer's disease (AD) is the most studied (Tapia-Rojas et al., 2019). The main hallmarks of AD pathology are intracellular deposition of tau neurofibrillary tangles and extracellular amyloid- $\beta$ (A $\beta$ ) plaques. Despite being initially considered as a pathological change driven by the toxic effects of amyloid peptide, our understanding of the role that tau plays in AD has been continuously evolving (Götz et al., 2019). Growing evidence indicates that tau pathology can also exert synergistic effects with amyloid peptide and that it correlates more closely to the progression and cognitive impairment of AD than A $\beta$ plaques (Bejanin et al., 2017; Guo et al., 2020). Additionally, given the failure of various clinical $A \beta$-directed therapies, more efforts have been focused on exploring tau-targeted therapies worldwide in recent years (Congdon and Sigurdsson, 2018). 
Phosphatase and tensin homolog (PTEN)-induced kinase 1 (PINK1), a serine/threonine kinase mainly localized to mitochondria, has attracted more and more attention since its mutation was identified in hereditary early-onset Parkinson's disease (PD) (Valente et al., 2004; Rasool and Trempe, 2018). PINK1 is widely distributed across multiple tissues and organs of the human body, with the brain being the region with the highest expression (Fagerberg et al., 2014). Under normal conditions, after being imported to the mitochondrial membrane, PINK1 is cleaved by mitochondrial presenilin-associated rhomboid-like (PARL) protease, after which it is transported into the cytoplasm, where it gets degraded. Upon mitochondrial depolarization, PINK1 is stabilized and activated on the mitochondrial membrane, and can initiate PINK1-Parkin dependent mitophagy (Arena and Valente, 2017). Beyond its originally perceived role as an initiator of mitophagy, PINK1 has also shown to be involved in regulating autophagy-lysosome pathway (ALP), ubiquitin-proteasome system (UPS), neurite outgrowth and neuron survival, inflammation, tumor suppression, and apoptosis (Michiorri et al., 2010; McLelland et al., 2014; Parganlija et al., 2014; Akabane et al., 2016; Arena and Valente, 2017; Sliter et al., 2018). In addition to PD, PINK1 has also been proven to exert neuroprotective effects in other neurodegenerative diseases, including Huntington's disease, amyotrophic lateral sclerosis, and AD (Khalil et al., 2015; Quinn et al., 2020; Baek et al., 2021).

Several studies have reported abnormal expression of PINK1 in patients with $\mathrm{AD}$ as well as in cellular and animal models of AD. Among them, most studies showed decreased levels of PINK1 in the context of AD pathology (Choi et al., 2014; Du et al., 2017; Manczak et al., 2018; Reddy et al., 2018; Fang et al., 2019; Ochi et al., 2020; Zhao et al., 2020; Liang et al., 2021), although there are also a minority of studies with opposing conclusions (Mise et al., 2017; Pakpian et al., 2020; Zheng et al., 2020). Researchers showed that the increasing expression of PINK1 lessened $A \beta$ plaques accumulation and rescued cognitive impairments in $\mathrm{AD}$ mice. The underlying mechanisms probably included induction of the autophagy pathway, altering APP transcription or secretases, increasing the phagocytosis of $\mathrm{A} \beta$ plaques by microglia, promoting mitophagy, and improving mitochondrial function (Du et al., 2017; Fang et al., 2019; Han et al., 2020). However, the specific impact of PINK1 on tau pathology remains largely underexplored and existing relevant and targeted studies are suggestive but inadequate. A previous study showed that activation of mitophagy reduced tau levels, while PINK1 knockdown abolished this effect (Fang et al., 2019). In a different study, G309D PINK1 mutation led to a significant increase in phosphorylated tau (Ser396/404) through inhibition of GSK3 $\beta$ activation in cells (Ye et al., 2015). More efforts are needed to establish the precise role of PINK1 in tau pathology and its possible underlying mechanisms.

Here, using a mouse model of tauopathy injected with AAV2-full-length human TAU into the hippocampus, we showed that upregulation of PINK1 significantly alleviated the deposition of pathological tau, neuron loss, synaptic damage, and cognitive impairments in mice. This occurred through inducing tau degradation via ALP, reducing tau accumulation in mitochondria and ameliorating mitochondrial disorders. Taken together, our study supports that PINK1 may be a promising target for $\mathrm{AD}$ treatment.

\section{MATERIALS AND METHODS}

\section{Animals}

Wild-type C57BL/6J mice (male, 8-10 weeks-age, 20-25 g) were acquired from Beijing Vital River Laboratory Animal Technology Co., Ltd. Animals were randomly assigned into cages (4-5 mice per cage), under normative cultured environment: 12-h day-night cycle with freely available food and water. All animal experiments were performed according to the "Policies on the Use of Animals and Humans in Neuroscience Research" revised and approved by the Society for Neuroscience in 1995, the Guidelines for the Care and Use of Laboratory Animals of the Ministry of Science and Technology of the People's Republic of China, and the Institutional Animal Care and Use Committee at Tongji Medical College. The animal study was reviewed and approved by Ethics Committee of Tongji Medical College, Huazhong University of Science and Technology.

\section{Stereotactic Brain Injection and Drug Administration}

pAAV-SYN-human Tau-mCherry-3×FLAG-WPRE $(1.30 \times$ $\left.10^{13} \mathrm{vg} / \mathrm{ml}\right), \quad$ pAAV-SYN-PINK1-EGFP-3×FLAG-WPRE $(1.35$ $\left.\times 10^{13} \mathrm{vg} / \mathrm{ml}\right)$, and corresponding vehicles pAAV-SYN-MCSmCherry-3 $\times$ FLAG $\left(2.09 \times 10^{13} \mathrm{vg} / \mathrm{ml}\right)$ and pAAV-SYN-MCSEGFP- $3 \times$ FLAG $\left(2.84 \times 10^{13} \mathrm{vg} / \mathrm{ml}\right)$ were generated by OBio Tech. Inc. (Shanghai, China). After being fixed on stereotaxic apparatus with adequate anesthesia, mice were injected with $1 \mu \mathrm{l}$ of virus into the hippocampal CA1 area bilaterally (AP-1.94, $\mathrm{ML} \pm 1.2, \mathrm{DV}-1.6)$. Injection rate was maintained at $100 \mathrm{nl} / \mathrm{min}$, and the needle syringe was kept in situ for an additional $10 \mathrm{~min}$ after the virus was fully injected. Before putting them back into cages, mice were placed on an electric blanket for revival.

Fourteen days after virus injection, mice were treated with (1) the autophagy inhibitor chloroquine (CQ) (C6628, SigmaAldrich) at $50 \mathrm{mg} / \mathrm{kg}$ body weight (Campos et al., 2020; Chen et al., 2020), (2) the proteasome inhibitor MG132 (M8699, SigmaAldrich) at $0.5 \mathrm{mg} / \mathrm{kg}$ body weight (Lu et al., 2017), or (3) the same volume of vehicle daily for 16 days via intraperitoneal injection.

\section{Behavior Tests}

One month after the stereotactic injection, behavioral experiments were conducted to evaluate the spatial learning and memory capabilities of the mice. The novel object recognition (NOR) test is a learning and memory evaluation method based on the principle that animals are born with a tendency to explore new things. This test was conducted as follows (Hong et al., 2020): $24 \mathrm{~h}$ before the test, mice were placed in the arenas $(50 \mathrm{~cm} \times 50 \mathrm{~cm}$ container $)$ 
without objects for a 5-min habituation. The next day, mice were put into the arenas (one sidewall with two identical objects $A$ and $A^{\prime}$ separately located on either end) for $5 \mathrm{~min}$. One hour later, object $A^{\prime}$ was replaced by a different object, object $B$, and then the animals were put into the arenas again and allowed to explore both objects for $5 \mathrm{~min}$. A video camera above the arenas logged the experimental behavior. The time that mice spent exploring object $A$ and object $B$ was recorded as $\mathrm{TA}$ and $\mathrm{TB}$, respectively. $\mathrm{TB} /(\mathrm{TA}+\mathrm{TB})$ was set as the recognition index.

The Morris water maze (MWM) test is used to assess the learning and memory abilities of laboratory animals in the context of spatial position and orientation (Morris, 1984). It was performed as follows: during the spatial learning phase, mice were trained to find a concealed platform in a fixed position below the waterline for five consecutive days. The training time was fixed at 12:00 pm-17:00 pm. In each training session, mice were gently put into water from one of the other three quadrants (without target platform), facing the pool wall. If the platform was not sought out within $60 \mathrm{~s}$, mice would be directed to the platform and made to stay in it for another $30 \mathrm{~s}$. On day 7, the platform was removed, and mice were put in the water maze for $60 \mathrm{~s}$ to test spatial memory. The motion trails of mice were recorded and analyzed using MWZ-100 system (Techman, China).

\section{Protein Extraction}

Hippocampal regions infected with virus were isolated and mechanically homogenized in lysis buffer for Western blotting (P0013, Beyotime). Homogenate was mixed with 8\% (wt/vol) SDS buffer and boiled for $10 \mathrm{~min}$. The sample was further disintegrated through sonication and centrifugation at $12,000 \times g$ for $15 \mathrm{~min}$ at $4^{\circ} \mathrm{C}$. Supernatant was collected as total protein extract.

For preparation of sarkosyl soluble/insoluble fractions (Goedert et al., 1992; Schlegel et al., 2019; Ferrer et al., 2020), the sample was mechanically homogenized in 10 volumes $(\mathrm{w} / \mathrm{v})$ of pre-cooling lysis buffer (10 mM Tris- $\mathrm{HCl}, \mathrm{pH} 7.4,0.8 \mathrm{M} \mathrm{NaCl}$, $1 \mathrm{mM}$ EGTA, 10\% sucrose) and then centrifuged at 20,000 $\times \mathrm{g}$ for $20 \mathrm{~min}$ at $4^{\circ} \mathrm{C}$. The supernatant (S1) was transferred to a new Eppendorf tube, and the pellet was re-homogenized in 5 volumes (w/v) of lysis buffer and spun at $20,000 \times g$ for $20 \mathrm{~min}$. The supernatant (S2) was mixed with supernatant (S1) and incubated with $1 \% \mathrm{~N}$-lauroylsarkosynate $(\mathrm{w} / \mathrm{v})$ for $1 \mathrm{~h}$ at room temperature while shaken. The sample was then spun at $100,000 \times g$ for $1 \mathrm{~h}$ at $4^{\circ} \mathrm{C}$. The supernatant was transferred to a new Eppendorf tube, designated as the soluble fraction. The pellet was re-suspended $(0.2 \mathrm{ml} / \mathrm{g})$ in $50 \mathrm{mM}$ Tris- $\mathrm{HCl}(\mathrm{pH} \mathrm{7.4)}$ and stored as sarkosyl insoluble fraction.

To separate out mitochondria from cytoplasm, we used the Tissue Mitochondria Isolation Kit (C3606, Beyotime). Following manufacturer's instructions, tissue sample was homogenized in solution $A$ and centrifuged at $1,000 \times g$ for $10 \mathrm{~min}$ at $4^{\circ} \mathrm{C}$. Supernatant was collected and centrifuged at $11,000 \times g$ for another $10 \mathrm{~min}$ at $4^{\circ} \mathrm{C}$. Then, supernatant was transferred to a new Eppendorf tube, designated as cytoplasm fraction. The deposit was re-suspended in a lysis buffer supplied by the kit and stored as mitochondrial fraction.

\section{Co-Immunoprecipitation}

Hippocampal regions infected with virus were isolated and mechanically homogenized in lysis buffer for immunoprecipitation (IP) (P0013, Beyotime). Then, the homogenate was centrifuged at $3,000 \mathrm{rpm}$, for $20 \mathrm{~min}$ at $4^{\circ} \mathrm{C}$. The supernatant was incubated with the primary antibody Tau5 $(2 \mu \mathrm{g} / 100 \mu \mathrm{g})(\mathrm{ab} 80579, \mathrm{Abcam})$ overnight at $4^{\circ} \mathrm{C}$ and then Protein $\mathrm{A}+\mathrm{G}$ Agarose $(30 \mu \mathrm{l} / 100 \mu \mathrm{l})$ (P2012, Beyotime) was added into the sample for $4-6 \mathrm{~h}$. After that, the agarose was washed three times. Proteins attached to the agarose were resuspended in buffer (50 mM Tris- $\mathrm{HCl}, \mathrm{pH} 6.8,2 \%$ SDS, $10 \%$ glycerol) and boiled for $10 \mathrm{~min}$. Collected protein sample was analyzed by Western blot.

\section{Western Blot}

Via SDS acrylamide gel electrophoresis, protein sample was transferred to nitrocellulose filter membrane (10600002, Whatman) and then blocked in 5\% skimmed milk for $1 \mathrm{~h}$. The membrane was then incubated with primary antibodies overnight at $4^{\circ} \mathrm{C}$ and then incubated with secondary antibody for $1 \mathrm{~h}$. Antibodies used in this study are listed in Table 1. Odyssey Infrared Imaging System (LI-COR Biosciences, Lincoln, NE, United States) and ECL Imaging System (610007-8Q, Clinx Science Instruments Co., Ltd.) were used for visualization of protein bands. Quantitative analysis of blots was performed using ImageJ software (Fiji) (Li et al., 2021).

\section{Quantitative Real-Time PCR}

Total RNA was isolated from virus-infected mice hippocampal region using Trizol reagent (15596018, Thermo Fisher Scientific). The transcription reagent kit (RR037, Takara) was then used for cDNA synthesis. Quantitative PCR was conducted using the One-Step SYBR PrimeScript PLUS RT-PCR Kit (RR096A, Takara) following the manufacturer's instructions. The PCR system contained $1 \mu \mathrm{l}$ of forward and reverse primers, $1 \mu \mathrm{l}$ of cDNA, $3 \mu \mathrm{l}$ of diethylpyrocarbonate (DEPC $\left.\mathrm{H}_{2} \mathrm{O}\right)$, and $5 \mu \mathrm{l}$ of SYBR Green PCR master mixes. RT-PCR was performed and analyzed using an ABI Step one plus Real-Time PCR System (Applied Biosystems). Primers for human TAU were F: $5^{\prime}$-CGCCAG GAGTTCGAAGTGAT-3' and R: $5^{\prime}$-TCTTGGTGCATGGTG TAGCC-3' (Korhonen et al., 2011) and primers for $\beta$-actin were F: $5^{\prime}$ - CAAATGTTGCTTGTCTGGTG-3' and R: $5^{\prime}$ GTCAGTCGAGTGCACAGTTT-3' (Wan et al., 2021).

\section{Immunohistochemical and Nissl's Staining}

Mice brain slices (paraffin section, $4 \mu \mathrm{m}$ thick) were baked at $55^{\circ} \mathrm{C}$ for $1 \mathrm{~h}$ and immersed into xylene for $40 \mathrm{~min}$. After being dewaxed, slices were dehydrated through graded ethanol $(100 \%, 100 \%, 95 \%, 90 \%$, and $80 \%)$ for $5 \mathrm{~min}$ each time. Brain slices were immersed into citric acid buffer $(\mathrm{pH}=$ $6.0,10 \mathrm{mM}$ ) and heated in a microwave for $10 \mathrm{~min}$ to maximize tissue antigen recovery. Then, slices were incubated with $3 \% \mathrm{H}_{2} \mathrm{O}_{2}$ for $30 \mathrm{~min}$ and blocked in $5 \%$ BSA solution containing $0.5 \%$ Triton X-100 for $40 \mathrm{~min}$. Next, slices were incubated with primary antibody (listed in Table 1) at $4^{\circ} \mathrm{C}$ for $24-48 \mathrm{~h}$. After incubation with 
TABLE 1 | Antibodies used in this study.

\begin{tabular}{|c|c|c|c|c|c|}
\hline Antibody & Host & $\begin{array}{c}\text { Dilution } \\
\text { WB }\end{array}$ & $\begin{array}{c}\text { Dilution } \\
\text { IHC }\end{array}$ & $\begin{array}{c}\text { Dilution } \\
\text { IF }\end{array}$ & Source \\
\hline Anti-PINK1 & Rabbit & $1: 500$ & & & BC100-494, Novus Biologicals \\
\hline Anti-PINK1 & Rabbit & $1: 500$ & & & ab23707, Abcam \\
\hline HT7 & Mouse & $1: 1,000$ & $1: 100$ & & MN1000, Thermo Fisher Scientific \\
\hline Tau5 & Mouse & $1: 1,000$ & & & ab80579, Abcam \\
\hline Anti-Tau (pS396) & Rabbit & $1: 1,000$ & $1: 100$ & & 11102, Signalway Antibody \\
\hline Anti-Tau (pS404) & Rabbit & $1: 1,000$ & $1: 100$ & & 11112, Signalway Antibody \\
\hline Anti-Tau (pT205) & Rabbit & $1: 1,000$ & & & 11108, Signalway Antibody \\
\hline Anti-GAPDH & Mouse & $1: 5,000$ & & & 60004-1-lg, Proteintech \\
\hline Anti-LC3B & Rabbit & $1: 1,000$ & & & ab51520, Abcam \\
\hline Anti-P62/SQSTM1 & Rabbit & $1: 1,000$ & & & 18420-1-AP, Proteintech \\
\hline Anti-LAMP2 & Mouse & $1: 1,000$ & & & 66301-1-lg, Proteintech \\
\hline Anti-Beclin1 & Rabbit & $1: 1,000$ & & & 11306-1-AP, Proteintech \\
\hline Anti-Ubiquitin & Mouse & $1: 1,000$ & & & sc-8017, Santa Cruz Biotechnology \\
\hline Anti-Parkin & Mouse & $1: 1,000$ & & & 4211S, Cell Signaling Technology \\
\hline Anti-COX IV & Rabbit & $1: 1,000$ & & & 11242-1-AP, Proteintech \\
\hline Anti-Caspase 3 & Rabbit & $1: 1,000$ & & & 9662S, Cell Signaling Technology \\
\hline Anti-Cleaved caspase 3 & Rabbit & $1: 500$ & & & 9661S, Cell Signaling Technology \\
\hline Anti-EGFP & Rabbit & & & $1: 100$ & GB11602, Servicebio \\
\hline Anti-lba1 & Mouse & & & $1: 100$ & GB12105, Servicebio \\
\hline Anti-mouse lgG & Goat & $1: 10,000$ & & & A23910, Abbkine \\
\hline Anti-rabbit lgG & Goat & $1: 10,000$ & & & A23920, Abbkine \\
\hline Anti-mouse lgG & Goat & $1: 3,000$ & & & A25012, Abbkine \\
\hline Anti-mouse lgG & Goat & $1: 5,000$ & & & SA00001-1, Proteintech \\
\hline Anti-rabbit lgG & Goat & $1: 5,000$ & & & SA00001-2, Proteintech \\
\hline Anti-NeuN & Rabbit & & $1: 200$ & & ab177487, Abcam \\
\hline Anti-NeuN & Mouse & & & $1: 200$ & ab104224, Abcam \\
\hline Anti-mouse lgG & Goat & & $1: 200$ & & G1216-3, Servicebio \\
\hline Anti-rabbit lgG & Goat & & $1: 200$ & & G1215-3, Servicebio \\
\hline Anti-rabbit lgG & Donkey & & & $1: 200$ & ANT024S, Antgene \\
\hline Anti-mouse lgG & Donkey & & & $1: 200$ & ANT029S, Antgene \\
\hline
\end{tabular}

WB, Western blot; IHC, immunohistochemical staining; IF, immunofluorescence staining.

secondary antibody at $37^{\circ} \mathrm{C}$ for $1 \mathrm{~h}$, slices were stained with $\mathrm{DAB}$ reagent (G1212, Servicebio). Then, slices were rehydrated through graded ethanol $(80 \%, 90 \%, 95 \%, 100 \%$, and $100 \%$ ) for 5 min each time, transparentized using xylene for $20 \mathrm{~min}$, and mounted with neutral balsam. For Nissl's staining, after deparaffinage and gradient alcohol dehydration, slices were washed with PBS for $3 \times 5 \mathrm{~min}$, and then dyed with $0.5 \%$ toluidine blue reagent ( $\mathrm{G} 1036$, Servicebio) for 2-5 min. If the slice was hyperchromatic, $0.1 \%$ glacial acetic acid was used for differentiation. Baked slices were mounted with neutral balsam. A scanning microscope (SV120, OLYMPUS) was used for imaging.

\section{Immunofluorescence Staining}

After aforementioned deparaffinage, dehydration, and antigen recovery, mice brain slices were washed with PBS for $3 \times 5 \mathrm{~min}$ and blocked in $5 \%$ donkey serum containing $0.5 \%$ Triton X-100 for $40 \mathrm{~min}$. Next, slices were incubated with primary antibody (listed in Table 1) at $4^{\circ} \mathrm{C}$ for $24-48 \mathrm{~h}$. After incubation with secondary antibody at $37^{\circ} \mathrm{C}$ for $1 \mathrm{~h}$, slices were washed with PBS for $3 \times 5$ min and stained with DAPI reagent (G1012, Servicebio) for $10 \mathrm{~min}$ at room temperature. Slices were sealed with anti-fluorescence quencher (G1401, Servicebio) and imaged using a scanning microscope (SV120, OLYMPUS).

\section{Golgi Staining}

FD Rapid GolgiStain ${ }^{\text {тм }}$ Kit (FD Neuro Technologies, PK401) was used for Golgi staining. After being deeply anesthetized, the brain of mice was removed and immersed in mixture solution $\mathrm{A}+\mathrm{B}(1: 1)$ for $2-4$ weeks. Then, the brain was transferred to solution $\mathrm{C}$ for 3-7 days, after which it was placed on an oscillating tissue slicer and cut into slices ( $100 \mu \mathrm{m}$ thick). After being air dried in the dark, the slices were stained with a mixture of solution $\mathrm{D}+\mathrm{E}+$ double distilled water (1:1:2) as per manufacturer's instructions. Images were taken using an optical microscope (Nikon, Japan).

\section{ATP Assay}

ATP levels were measured using the ATP bioluminescence detection kit (S0026, Beyotime). Briefly, the hippocampal regions infected with virus was extracted and pyrolyzed with a lysis buffer supplied with the kit. The homogenate was centrifuged at $12,000 \times g$ for $5 \mathrm{~min}$ at $4^{\circ} \mathrm{C}$. Supernatant was collected for ATP detection. Protein concentration of the supernatant was measured using the BCA Protein Assay Kit (P0012S, Beyotime). Furthermore, $100 \mu \mathrm{l}$ of supernatant and $100 \mu \mathrm{l}$ of ATP detection buffer were mixed and the luminescence was measured using a microplate reader. Gradient dilution of the standard solution was conducted to 
generate the standard curve $(1 \mathrm{nM}-1 \mu \mathrm{M})$. ATP levels were calculated according to the standard curve and normalized against the standards' protein concentration.

\section{Malondialdehyde Assay}

Malondialdehyde (MDA) levels were measured using the Lipid Peroxidation MDA Assay Kit (S0131S, Beyotime). As per manufacturer's instructions, hippocampal regions infected with virus was extracted and pyrolyzed in RIPA lysis buffer (P0013D, Beyotime) and then centrifuged at $12,000 \times g$ for $10 \mathrm{~min}$ at $4^{\circ} \mathrm{C}$. Supernatant was collected for MDA detection. Protein concentration of the supernatant was measured using a BCA Protein Assay Kit (P0012S, Beyotime). One hundred microliters of supernatant was mixed with $200 \mu$ l of MDA detection working buffer containing thiobarbituric acid (TBA) and the MDA-TBA adduct was measured using a microplate reader at $535 \mathrm{~nm}$. Gradient dilution of standard solutions was conducted to generate the standard curve $(1-100 \mu \mathrm{M})$. MDA levels were calculated according to the standard curve and normalized against the standards' protein concentration, shown as nmol/ mg protein.

\section{Statistical Analysis}

All data were collected and analyzed in a blinded manner. Data were shown as mean \pm SEM or mean \pm SD and analyzed using GraphPad Prism (GraphPad Software, Inc., La Jolla, CA, United States). Statistical analyses were conducted using twotailed unpaired $t$-tests, one-way ANOVA, or two-way repeated measures ANOVA followed by Tukey multiple-comparisons post-hoc tests. $p<0.05$ was set as the level of statistical significance.

\section{RESULTS}

\section{PINK1 Rescues Cognitive Impairments in hTau Mice}

We injected pAAV-SYN-human Tau-mCherry-3×FLAGWPRE into the hippocampal CA1 region of mice for 1 month to mimic Alzheimer-like deposits of tau in the brain (Andorfer et al., 2003; Lasagna-Reeves et al., 2011; Li et al., 2019; Wan et al., 2021). Meanwhile, pAAV-SYNPINK1-EGFP-3 $\times$ FLAG-WPRE was also co-injected to explore its effect on tau pathology. SYN is a neuronspecific promoter, which means exogenous PINK1 and hTau would be specifically expressed in neurons. A high transfection efficiency of the virus was confirmed by Western blot, immunofluorescence and immunohistochemistry (Figures 4A,B,E; Supplementary Figure S1). As core symptoms of $\mathrm{AD}$, cognitive decline and dementia are tightly associated with tau pathology (Bejanin et al., 2017). Thus, we conducted behavioral tests on the mice to assess cognitive function (Figure 1A). In contrast with WT mice, human Tau (hTau) mice (injected with pAAV-SYN-human TAU-mCherry-3×FLAG-WPRE) showed obvious learning and memory impairments as evaluated by NOR and MWM tests, while overexpression of PINK1 rescued the cognitive dysfunction. More specifically, in the NOR test, the time that hTau mice spent on exploring novel object was significantly reduced; however, this recognition index was improved by PINK1 overexpression (Figures 1B-D). In the MWM test, compared with the WT group, hTau mice had a longer latency period before finding the hidden platform at 3rd-5th days during the training stage. During the test stage, longer latency to reach the place where the platform was previously placed before, less retention time in the target quadrant, and fewer times crossing the platform region were observed in hTau mice, while overexpressing PINK1 attenuated the above learning and memory deficits, as evidenced by decreased time to find the platform during the 4 th and 5 th day during the training phase, less escape latency, more retention time in the target quadrant, and more times crossing the platform region during the test phase (Figures 1E-I). There was no significant difference in swimming speed among the four groups of mice (Figure 1J), which excluded defects in motor ability. Overall, our data demonstrated that PINK1 overexpression ameliorates cognitive deficits in hTau mice.

\section{PINK1 ameliorates hTau-Induced Neuron Loss and Synaptic Damage}

Growing evidence supports the neurotoxic effects of tau as a primary event for neuron loss and synaptic injury, both of which are common neuropathologic manifestations in $\mathrm{AD}$ and closely related to the severity of cognitive disfunction (Iqbal and Grundke-Iqbal, 2002; Giannakopoulos et al., 2003). Especially, neuron loss in the hippocampal CA1 region is a prominent feature of AD (West et al., 1994; Simic et al., 1997). Therefore, we aimed to investigate the underlying mechanisms by which PINK1 ameliorated cognitive deficits in hTau mice. Nissl's staining and immunohistochemical staining were used to observe and compare neuron morphology and number in the hippocampal CA1 area of mice. In WT mice, irrespective of whether injected with PINK1 or not, neurons in the CA1 region had a full and orderly shape and were closely arranged with the Nissl bodies. Meanwhile, hTau mice displayed a reduced number of intact neurons (Figures 2A,B). Neurons had abnormal morphology, with an obscure structure and disorganized arrangement (Figure 2A). Interestingly, this phenotype was noticeably attenuated following PINK1 overexpression (Figures 2A,B). NeuN staining data also supported the finding that PINK1 overexpression ameliorated the neuron loss induced by accumulation of hTau (Figures 2C,D). In addition, overexpression of PINK1 attenuated the observed increased levels of cleaved caspase- 3 induced by hTau, thus suggesting that PINK1 alleviates hTau-induced cell apoptosis (Supplementary Figure S2).

The dendritic spine, functional protrusions on dendrite branches, is the main site of synaptogenesis, and so is closely related to synaptic transmission (Chidambaram et al., 2019). 


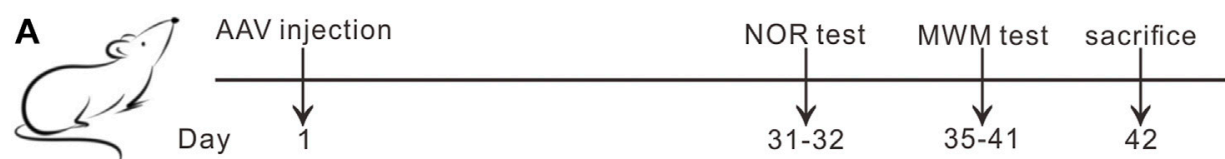

B

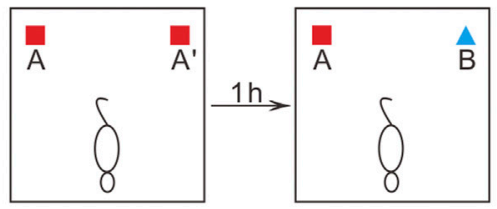

E
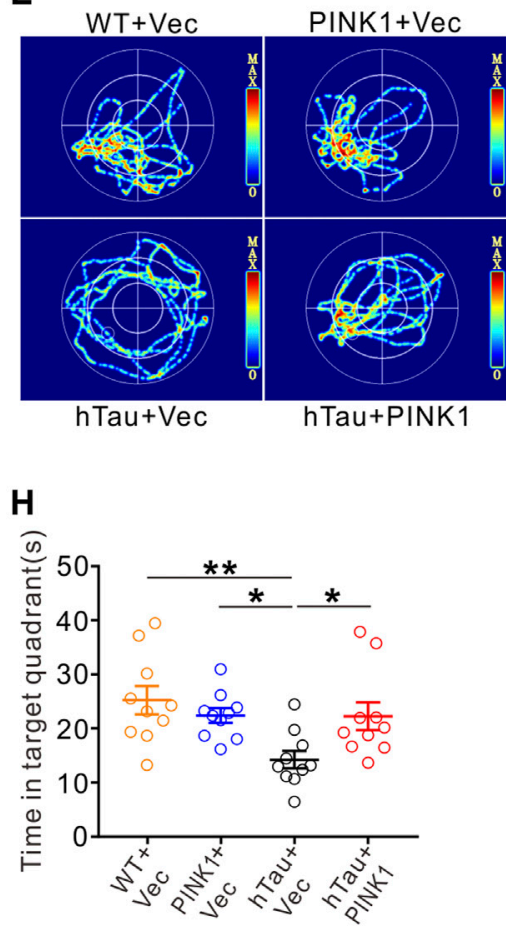

C

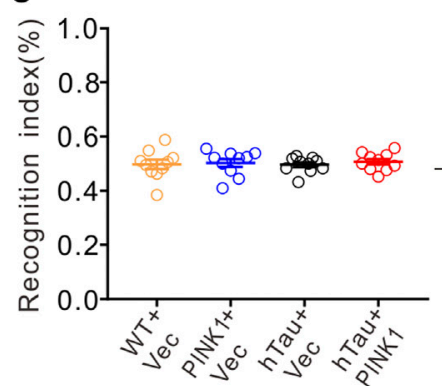

F

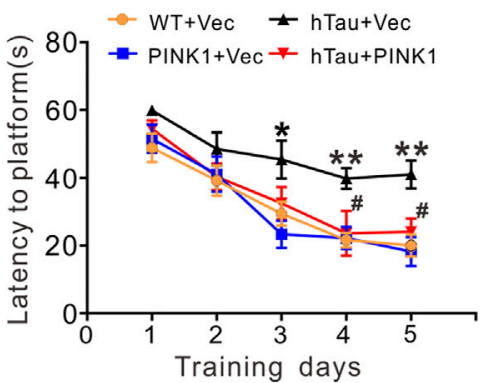

\section{I}

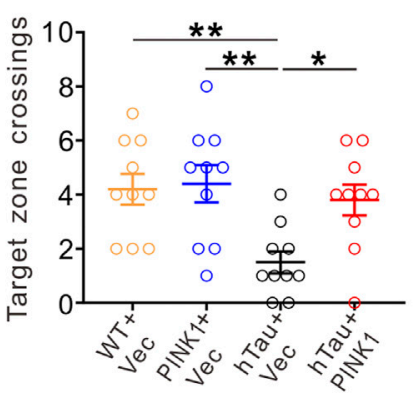

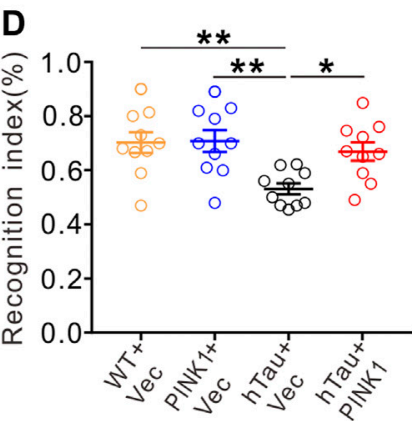

G

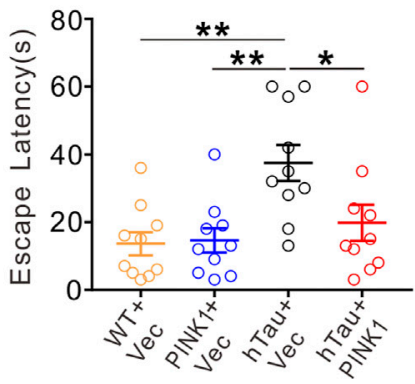

\section{J}

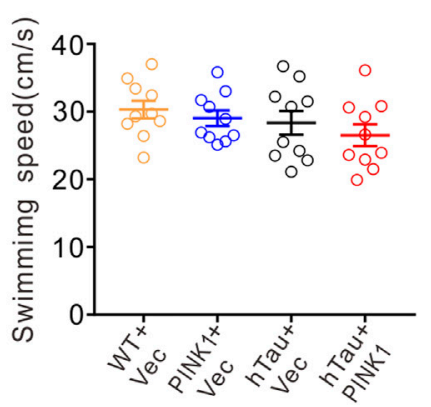

FIGURE 1 | PINK1 ameliorates cognitive impairments in hTau mice. (A) Experimental processes of virus injection and behavioral tests. (B-D) PINK1 improved cognitive performance of hTau mice in the NOR test shown by elevated recognition index. One-way ANOVA followed by Tukey multiple-comparisons tests. ${ }^{\star} p<0.05,{ }^{* *} p<0.01$. (E) Representative swimming path of mice in each group during the MWM probe test. (F) PINK1 improved learning ability in hTau mice shown by shortened latency to find the hidden platform during training stage in the MMW test. Two-way repeated-measures ANOVA followed by Tukey multiple-comparisons tests. ${ }^{*} p<0.05,{ }^{\star *} p<0.01 \mathrm{vs}$. WT + Vec; ${ }^{*} p<0.05$ vs. hTau + Vec. (G-I) PINK1 improved memory ability in hTau mice shown by decreased latency to reach the location of platform (G), longer retention time in the target quadrant (H), and more target zone crossings (I) during the MWM probe test. One-way ANOVA followed by Tukey multiple-comparisons tests. ${ }^{\star} p<0.05,{ }^{* *} p<0.01$. (J) No significant difference in swimming speed was seen among the four groups during the MWM probe test. One-way ANOVA followed by Tukey multiple-comparisons tests. All data were presented as mean \pm SEM. $n=10$ mice for each group.

Here, we identified and quantified dendritic spines in the CA1 region using Golgi staining. Compared with WT mice, dendritic branches were sparser, and the dendritic spine density was significantly reduced in hTau mice (Figure 3).
Meanwhile, overexpression of PINK1 attenuated such phenotype in hTau mice (Figure 3). These data indicated that PINK1 attenuates hTau-mediated neuron loss and synaptic damage. 

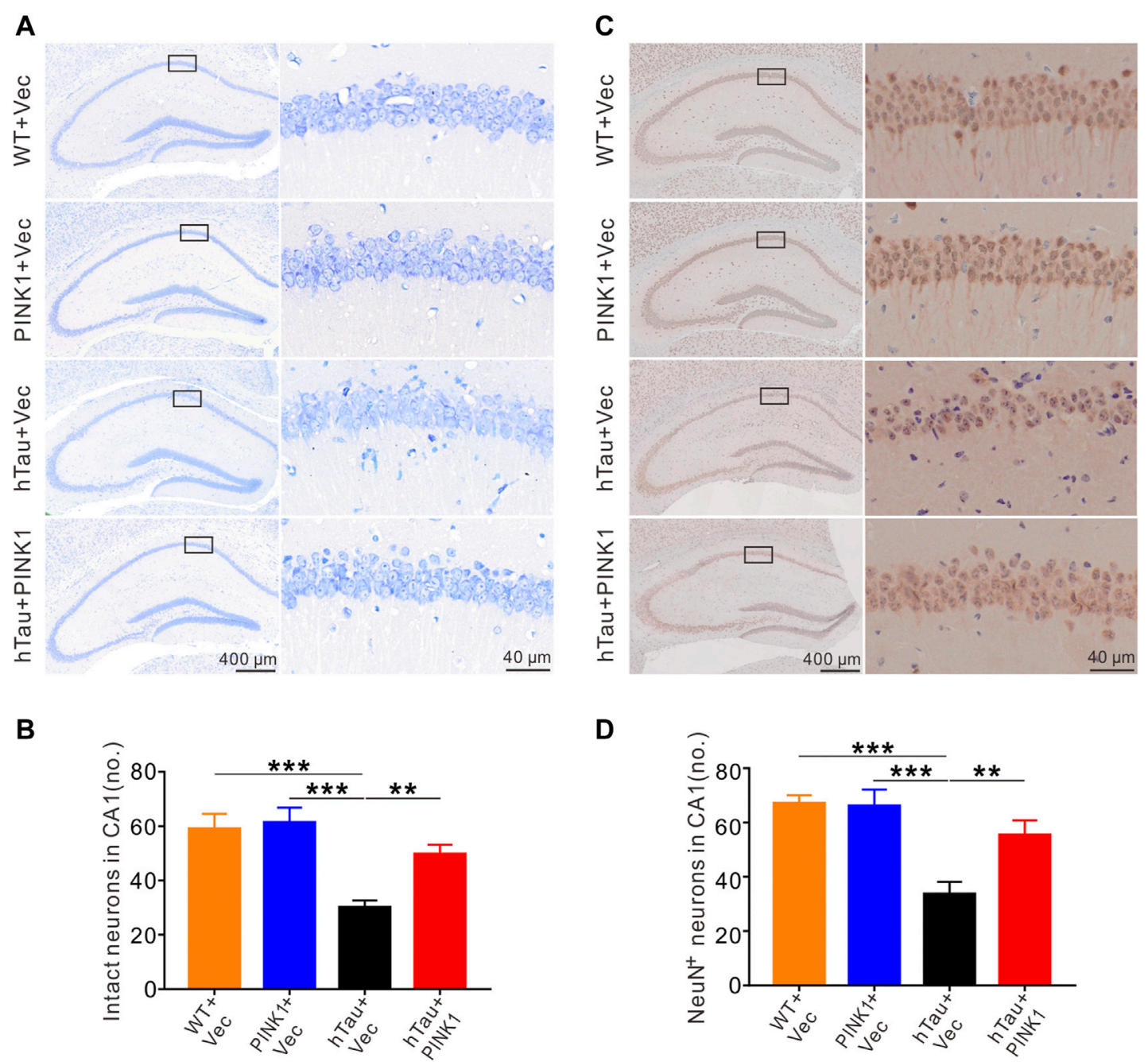

FIGURE 2 |PINK1 alleviates hTau-induced neuronal loss in the hippocampal CA1 region of mice. (A) PINK1 ameliorated hippocampal CA1 neuronal loss in hTau mice exhibited by representative images of Nissl staining. (B) Quantitative analysis of numbers of intact neurons in area framed within black bordered rectangle. Neurons with visible nuclei, distinctive nucleolus, and cytoplasmic Nissl staining were regarded as intact neurons and counted. One-way ANOVA followed by Tukey multiplecomparisons tests. ${ }^{\star *} p<0.01,{ }^{\star \star *} p<0.001$. (C) Representative images of NeuN immunohistochemical staining. (D) Quantitative analysis of numbers of neurons with positive NeuN staining in area framed within black bordered rectangle. One-way ANOVA followed by Tukey multiple-comparisons tests. ${ }^{\star \star} p<0.01$, ${ }^{\star \star \star} p<0.001$. All data were presented as mean \pm SD. $n=3$ mice for each group.

\section{PINK1 Overexpression Reduces Tau Protein Levels in hTau Mice}

We observed obvious accumulation of exogenous tau (human Tau) proteins in the hippocampal CA1 region of hTau mice, including total human tau (detected using the HT7 and Tau5 antibodies) and phosphorylated tau at Ser396, Ser404, and Thr205 (Figures 4A,B,E), as detected by Western blot or immunohistochemistry. Simultaneously overexpressing PINK1 significantly reduced the levels of exogenous total and phosphorylated tau proteins (Figures $4 \mathbf{A}, \mathbf{B}, \mathbf{E}$ ). Furthermore, we found that PINK1 overexpression decreased soluble and insoluble exogenous total and phosphorylated tau proteins compared with hTau mice (Figures 5E-H, 1-3 lane vs. 4-6 lane). PINK1 did not alter the mRNA levels of hTau (Figure 4D), which suggested that overexpressing PINK1 decreased hTau protein levels as a result of an increase in its degradation. Although the endogenous levels of mouse tau displayed a downward trend in the context of PINK1 overexpression, there was no significant difference among the four groups (Figures $4 \mathrm{~A}, \mathrm{C}$ ). Overall, these data showed that PINK1 decreases the pathological accumulation of tau proteins induced by hTau overexpression.

\section{PINK1 Promotes Clearance of hTau Accumulation via the Autophagy-Lysosome Pathway}

Given the protective effects of PINK1 against abnormally accumulated tau protein in mice, we sought to find out the 

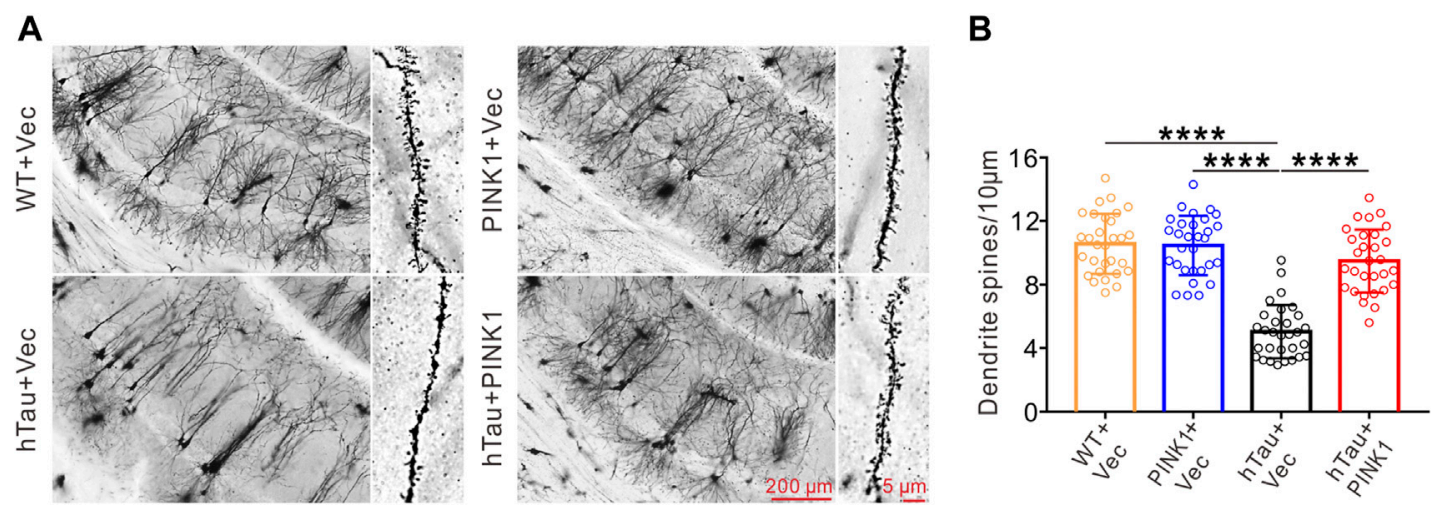

FIGURE 3 PINK1 reverses the decreased dendritic spine density in the hippocampal CA1 region of hTau mice. (A) Representative images of Golgi Staining in the hippocampal CA1 region of mice. (B) Quantitative analysis of spine density in the CA1 area of mice. Thirty neurons from each group were analyzed. One-way ANOVA followed by Tukey multiple-comparisons tests. ${ }^{* \star *} p<0.0001$. All data were presented as mean \pm SD. $n=3$ mice for each group.

potential underlying mechanism. As PINK1 did not lead to changes in the mRNA levels of hTau (Figure 4D), we inferred that PINK1 may affect the degradation pathways of hTau proteins. It has been previously shown that both UPS and ALP contribute to the degradation of tau aggregation in $\mathrm{AD}$ (Cheng et al., 2018), and there are also data that indicate the promotive effects of PINK1 on ALP (Michiorri et al., 2010; Parganlija et al., 2014; Du et al., 2017). Therefore, we aimed to detect the expression of autophagic markers (Abdelfatah et al., 2021). As illustrated in Figures 5A,B, PINK1 overexpression induced an increase in the expression of LC3 II and lysosomal protein LAMP2, and a decrease in the levels of $\mathrm{p} 62$ in the hippocampal CA1 region of hTau mice. The level of Beclin1 was not significantly altered among the four groups. Thus, these results suggest that PINK1 activates ALP in hTau mice.

To further confirm the pathway by which PINK1 induces elimination of abnormal accumulation of tau, MG132, an inhibitor of proteasome pathway, or chloroquine (CQ), an inhibitor of autophagy that blocks the fusion of autophagosome and lysosome, was used to treat the mice overexpressing hTau and PINK1, respectively. We found that CQ (lanes 10-12) but not MG132 treatment (lanes 7-9) reversed the decreased total (Tau5) and phosphorylated tau (pS396, pS404, and pT205) levels induced by PINK1 (Figures 5C,D; Supplementary Figure S3). Soluble and insoluble proteins were extracted, and total or phosphorylated tau levels were detected by Western blot. The levels of total and phosphorylated tau in the soluble and insoluble fraction of hTau mice were decreased following PINK1 overexpression, while CQ reversed the PINK1-induced reduction of total or phosphorylated tau levels (Figures 5E-H, lanes 10-12 vs. lanes 4-6; Supplementary Figure S3). MG132 treatment merely induced a small increase in total and phosphorylated tau levels in the soluble fraction, but had no effects in the levels of total or phosphorylated tau in the insoluble fraction (Figures 5E-H, lanes 7-9 vs. lanes 4-6; Supplementary Figure S3). We also observed that CQ caused an increase in the levels of endogenous tau (Figures 5E,F; Supplementary Figures S4A,B). All these data suggest that PINK1 decreases the levels of tau through the autophagy pathway.

\section{CQ Reverses the Improved Effects of PINK1 on Cognition}

Furthermore, we conducted behavioral experiments to investigate whether the improved cognitive function induced by PINK1 was also reversed by CQ treatment (Figure 6A). Unsurprisingly, we found that CQ treatment caused a distinct cognitive decline in hTau and PINK1 overexpressing mice. This was evidenced by a lower recognition index in the NOR test (Figures 6B,C), a longer latency period to reach the hidden platform on days 3-5 during the training phase, as well as a longer escape latency, shorter residence time in the target quadrant, and decreased platform zone crossing times during the test phase of the MWM test (Figures 6D-I). Treatment with MG132 did not induce significant changes in the cognition of hTau and PINK1 overexpressing mice (Supplementary Figure S5).

\section{PINK1 Reduces the Accumulation of hTau in Mitochondria and Improves Mitochondrial Function}

Besides our above discovery that PINK1 reduces tau proteins through the autophagy pathway, PINK1 has been widely reported to play vital roles in maintaining mitochondrial homeostasis (Arena and Valente, 2017). Here, we extracted the mitochondrial and cytoplasmic fraction from the hippocampal CA1 region of mice, and found accumulation of hTau in the mitochondrial fraction of hTau mice, which is consistent with our previous study in cells overexpressing hTau (Hu et al., 2016) as well as other previous studies (Lasagna-Reeves et al., 2011; Grassi et al., 2019; Torres et al., 2021), Meanwhile, PINK1 overexpression decreased the levels of hTau in both the mitochondrial and cytoplasmic fraction of hTau mice (Figures 7A,B). Furthermore, we 


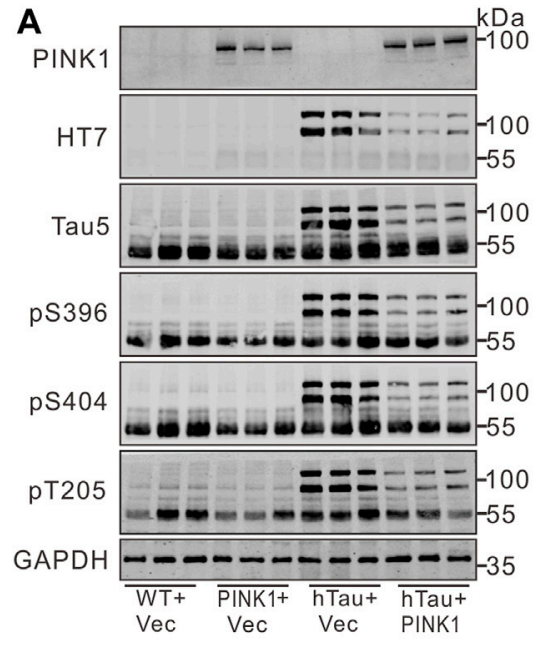

E

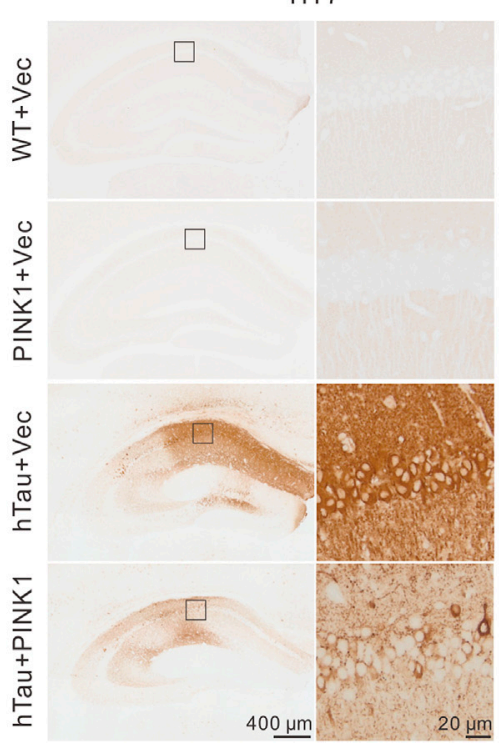

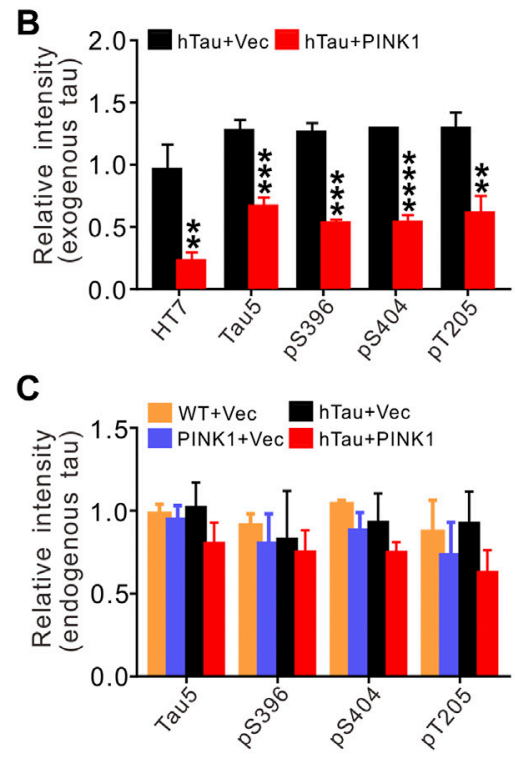

D

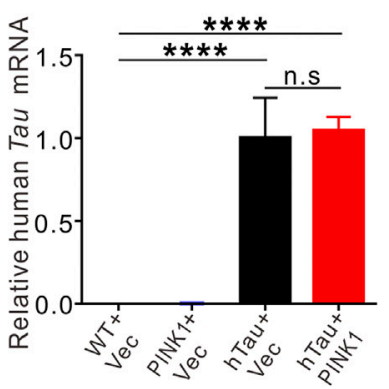

pS396

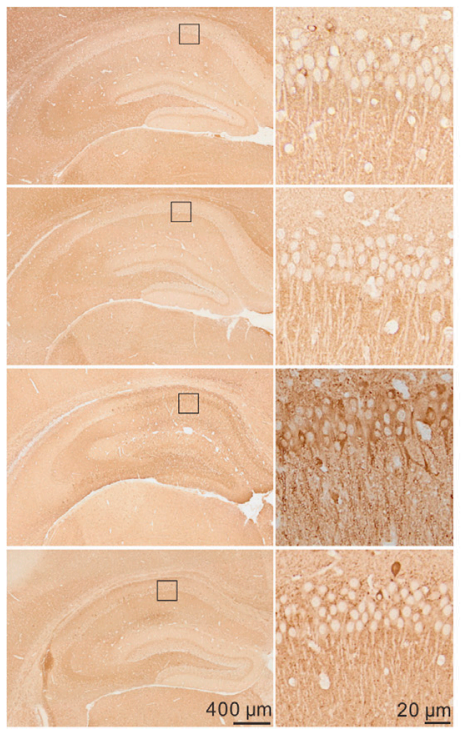

pS404

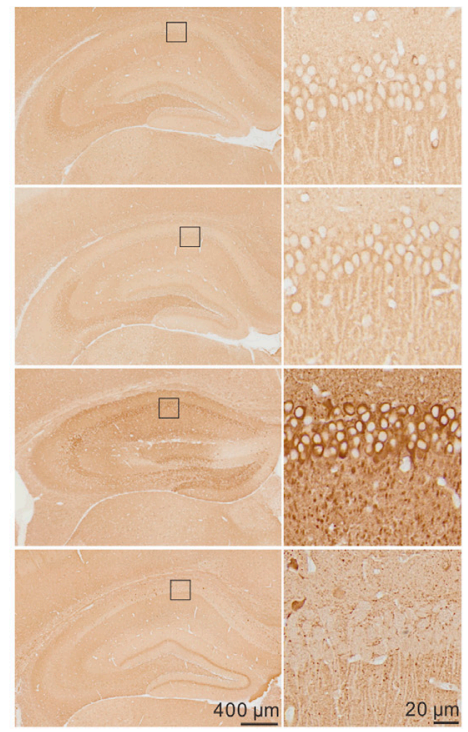

FIGURE 4 | Overexpression of PINK1 decreases tau protein levels in hTau mice. (A,B) Representative images and quantitative analysis of Western blot showed overexpression of PINK1 diminished levels of exogenous tau ( 106 kDa, human tau), including total tau (HT7, Tau5) and phosphorylated tau (pS396, pS404, and pT205) in the hippocampal CA1 area of hTau mice. Unpaired $t$-tests. ${ }^{\star \star} p<0.01,{ }^{\star \star \star} p<0.001,{ }^{\star \star \star \star} p<0.0001$. (C) Significant alteration of endogenous tau ( 55 kDa) was not found among the four groups. One-way ANOVA followed by Tukey multiple-comparisons tests. (D) The levels of human TAU mRNA had no significant changes with PINK1 overexpression. One-way ANOVA followed by Tukey multiple-comparisons tests. ${ }^{\star \star \star \star} p<0.0001$. (E) Representative images of immunohistochemical staining showed PINK1 reduced tau pathology (total tau, phosphorylated tau at Ser396 or Ser404) in the hippocampal CA1 area. HT7 antibody exclusively reacts to human tau proteins. All data were presented as mean \pm SD. $n=3$ mice for each group.

found reduced levels of Parkin in the mitochondrial and cytoplasmic fraction of mice with PINK1 overexpression (Figures 7A,C), indicating an activation of Parkin by PINK1 as activation of Parkin would induce its own ubiquitylation and degradation (Zhang et al., 2000; Xiong et al., 2009; McWilliams et al., 2018). In line with previous studies (Li et al., 2016; Guha et al., 2020; Szabo et al., 2020), assessment of mitochondrial function revealed mitochondrial dysfunction in hTau mice, as evidenced by reduced levels of ATP and elevated MDA levels, both of which were reversed in the context of PINK1 overexpression (Figures 7D,E).

\section{DISCUSSION}

As a primary cause of dementia and death in older people, AD has become a common problem and challenge in an aging 
A

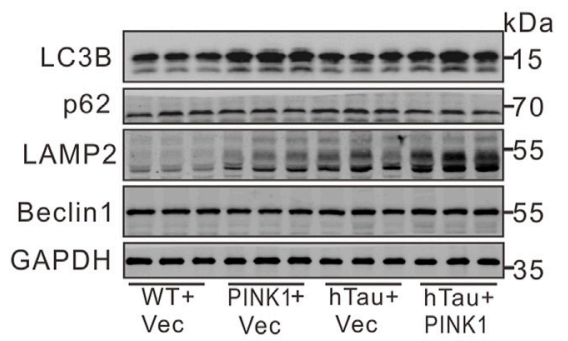

C

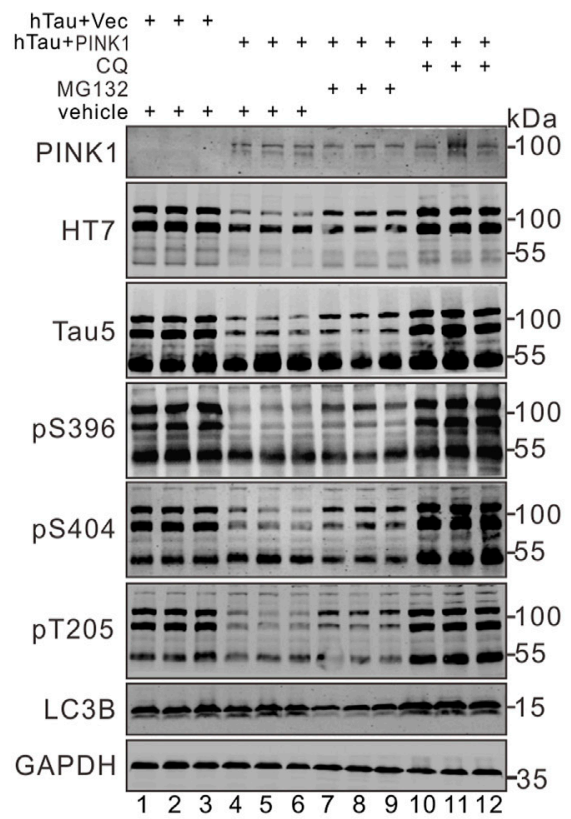

B $\quad$ WT+Vec $\square_{\text {TTau+Vec }}$

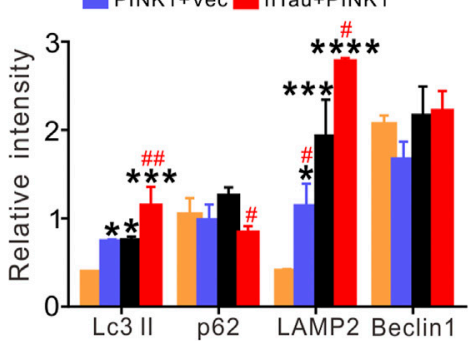

D

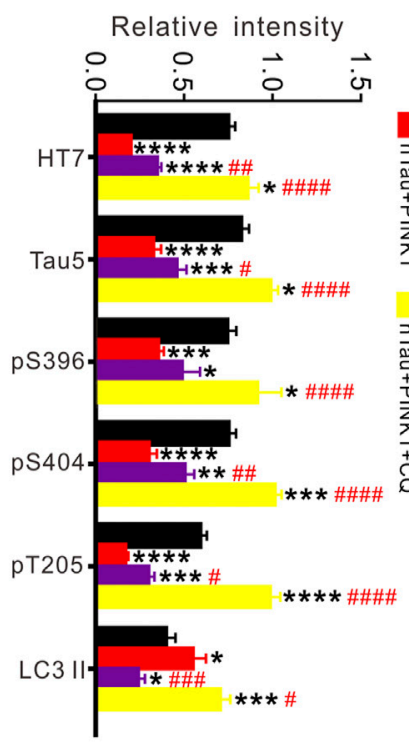

Soluble

E

hTau+Vec + + +

hTau+PINK1 $1++++++++++$

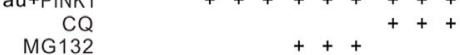

vehicle $++++++++\mathrm{KDa}^{+}$

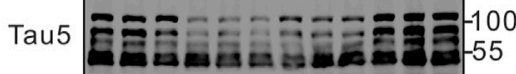

pS396 ミミーーーーーシーミミー-100

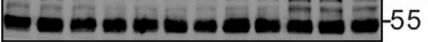

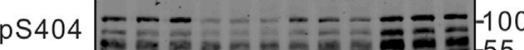

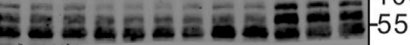

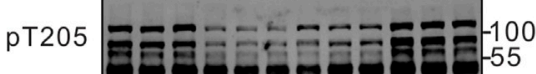

GAPDH - - - - -

$122 \quad 3 \quad 4 \quad 5 \quad 6 \quad 7 \quad 899101112$

$F$

Insoluble

$\underset{\mathrm{hTau}+\mathrm{Vec}}{\mathrm{hTau+PINK} 1}+++++++++++$

hTau+PINK1
CQ
++++
++++

MG132

vehicle $++++++{ }^{+}+\mathrm{kDa}$

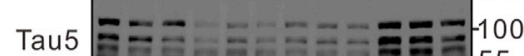

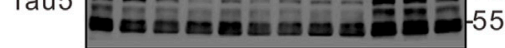

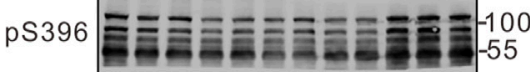

pS404 ニニニニニニニニニニニニ-100

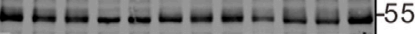

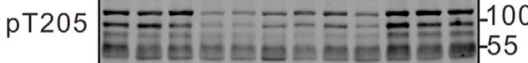

GAPDH

G exogenous tau in soluble fraction

H exogenous tau in insoluble fraction
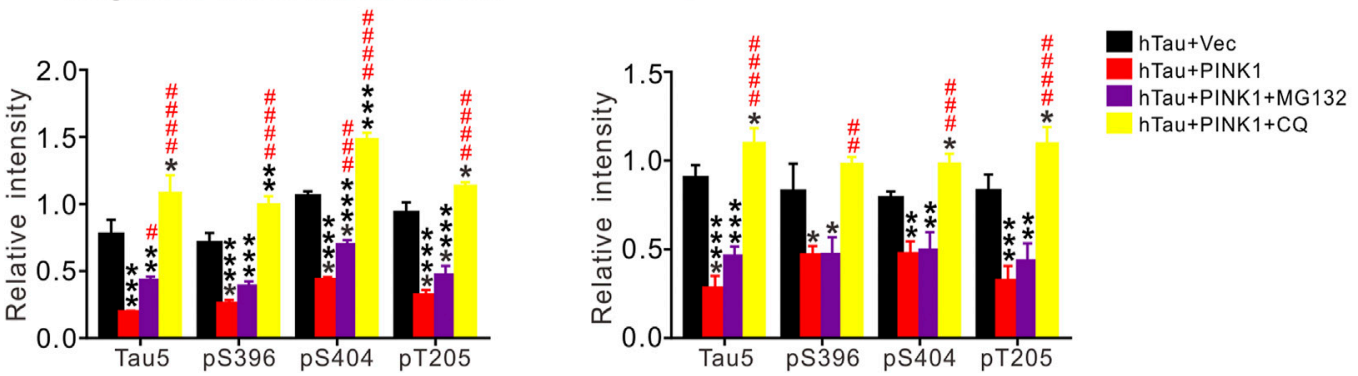

FIGURE 5 |PINK1 promotes clearance of hTau mainly through the autophagy pathway. (A,B) PINK1 increased the levels of LC3 II and lysosomal protein LAMP2, as well as decreased p62 levels detected by Western blot. One-way ANOVA followed by Tukey multiple-comparisons tests. ${ }^{\star} p<0.05,{ }^{\star \star} p<0.01$, ${ }^{* \star *} p<0.001$, ${ }^{\star \star \star \star *} p<0.0001$ vs. WT + Vec; ${ }^{\#} p<0.05,{ }^{\# \#} p<0.01$ vs. hTau + Vec. (C,D) In total fraction of mice hippocampal CA1, PINK1 diminished the levels of exogenous tau ( 106 $\mathrm{kDa}$, human tau: both total and phosphorylated tau), while CQ treatment reversed this reduction shown by Western blot. Treatment with MG132 induced relatively small increase in the levels of hTau proteins in hTau and PINK1 overexpressing mice. (E-H) In both the soluble $\mathbf{( E ,} \mathbf{G})$ and insoluble fraction $(\mathbf{F}, \mathbf{H})$ of mice hippocampal CA1 area, PINK1 decreased the levels of exogenous tau ( 106 kDa, human tau: both total and phosphorylated tau) in hTau mice, while CQ treatment reversed the reduction. Treatment with MG132 induced relatively small increase in the levels of hTau proteins in the soluble fraction. One-way ANOVA followed by Tukey multiple-

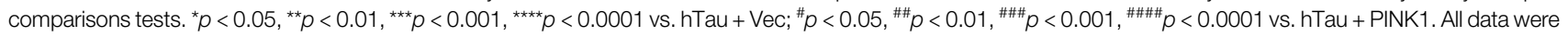
presented as mean \pm SD. $n=3$ mice for each group. 


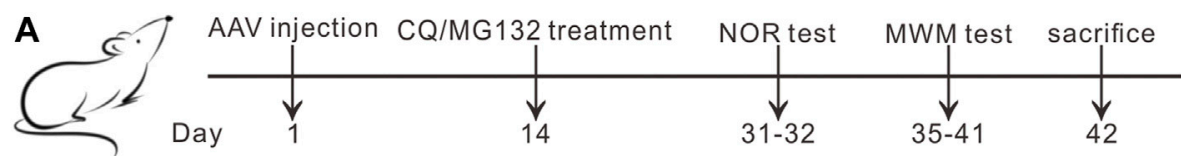

B

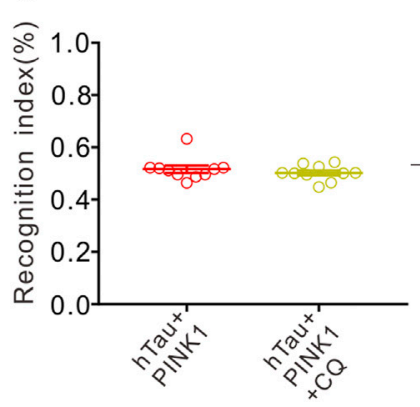

E
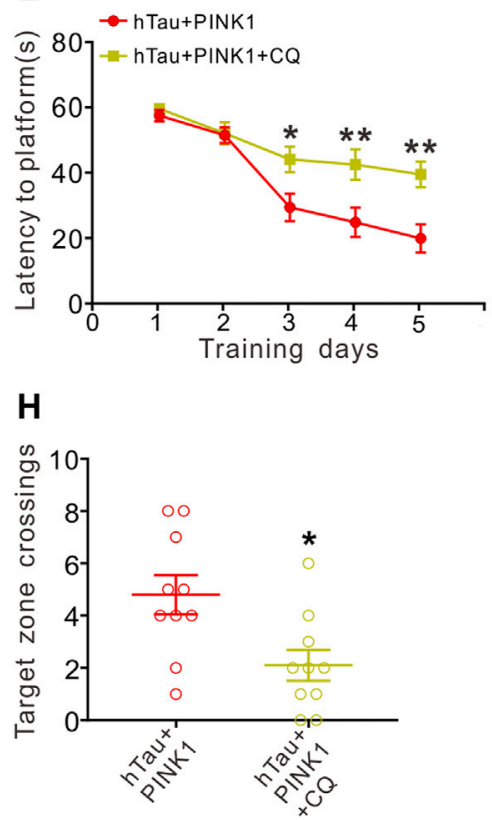

C

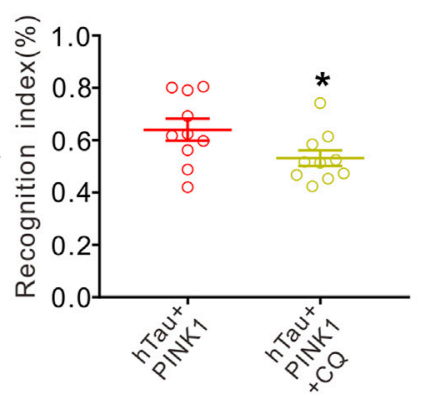

F

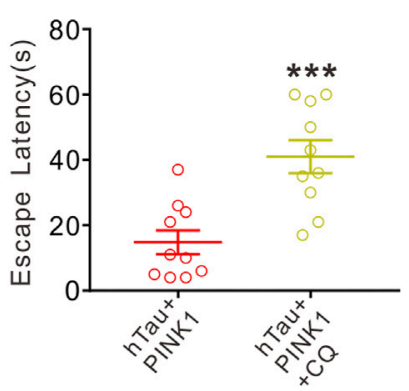

I

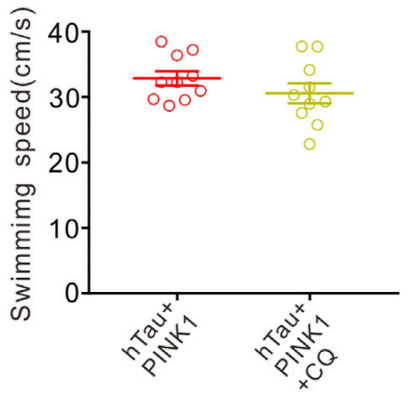

D

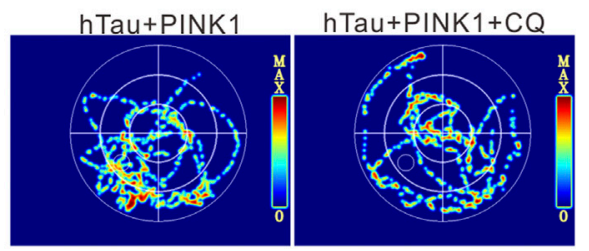

G

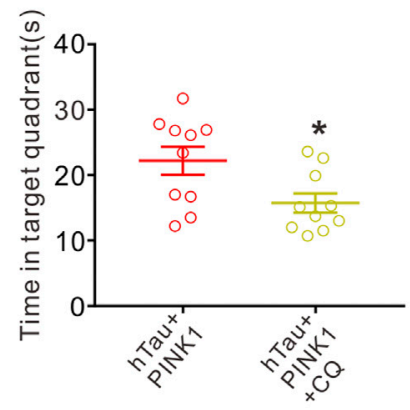

FIGURE 6 | CQ treatment reverses the improved cognition induced by PINK1 overexpression. (A) Experimental processes of virus injection, drug treatment, and behavioral tests. (B,C) CQ treatment lowered the recognition index of hTau and PINK1 overexpressing mice in the NOR test. Unpaired $t$-tests. ${ }^{*} p<0.05$. (D) Representative swimming path of mice in each group during the MWM probe test. (E) CQ treatment impaired the learning ability of hTau and PINK1 overexpressing mice, shown by prolonged latency to find the hidden platform during training stage in the MWM test. Two-way repeated-measures ANOVA followed by Tukey multiplecomparisons tests. ${ }^{\star} p<0.05,{ }^{\star *} p<0.01$. (F-H) CQ treatment impaired the memory ability of hTau and PINK1 overexpressing mice, shown by longer latency to reach the location of platform (F), shorter retention time in the target quadrant (G) and fewer target zone crossings $(\mathbf{H})$ during the MWM probe test. Unpaired $t$-tests. ${ }^{*} p<0.05$, ${ }^{\star \star} p<0.01,{ }^{\star \star \star} p<0.001$. (I) No significant difference in swimming speed was seen between the two groups during the MWM probe test. Unpaired $t$-tests. All data were presented as mean \pm SEM. $n=10$ mice for each group.

society due to a lack of effective diagnosis and treatment. Here, we injected AAV2-full-length human TAU into the hippocampal CA1 region of mice to mimic Alzheimer-like tau pathology in the brain. We observed an obvious accumulation of tau proteins, neuron loss, synapse injury, mitochondrial function disorders, and cognitive impairments in hTau mice. We also found that overexpression of PINK1 effectively reduced neuropathological accumulation of tau proteins, ameliorated mitochondrial function, attenuated damage to neurons and synapses, and thus rescued cognitive decline in hTau mice.

In our study, overexpression of PINK1 led to activation of ALP, as evidenced by increased LC3 II and lysosomal protein LAMP2, as well as decreased levels of p62. PINK1-induced ALP activation has also been observed in other studies (Michiorri et al., 2010; Parganlija et al., 2014; Du et al., 2017). In transgenic mAPP mice, overexpression of PINK1 increased the expression of LC3 II, lysosome-associated 

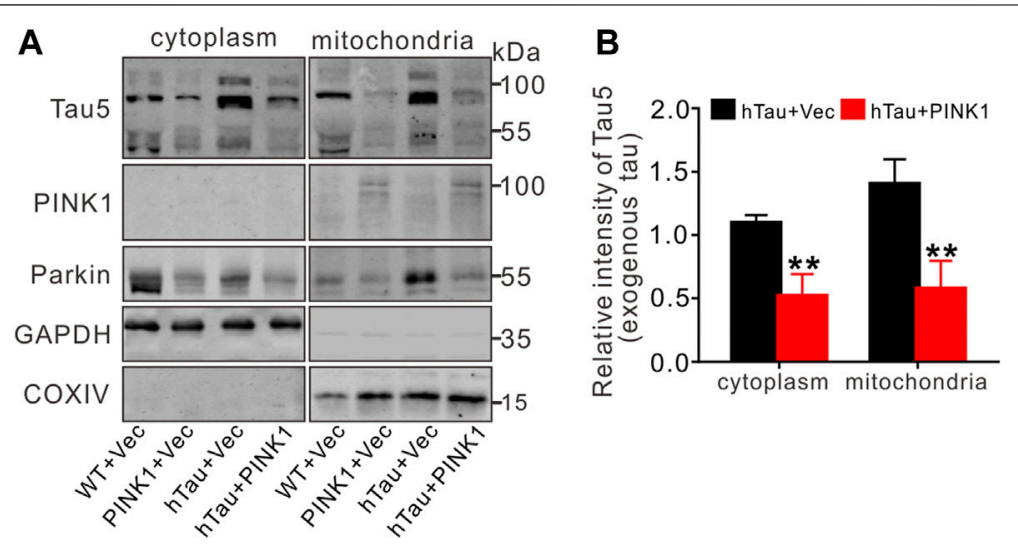

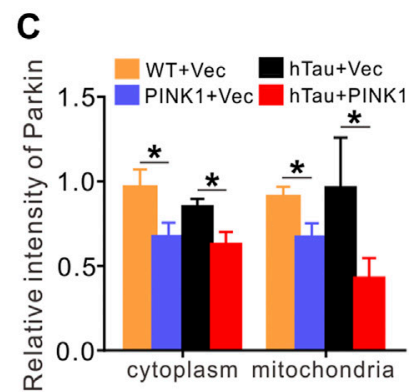

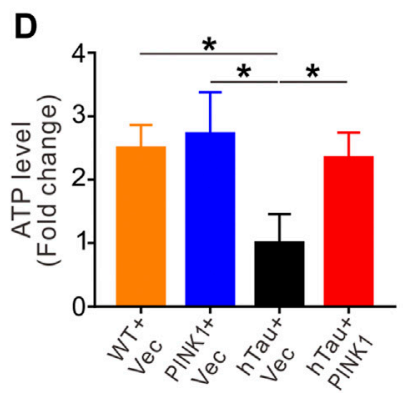

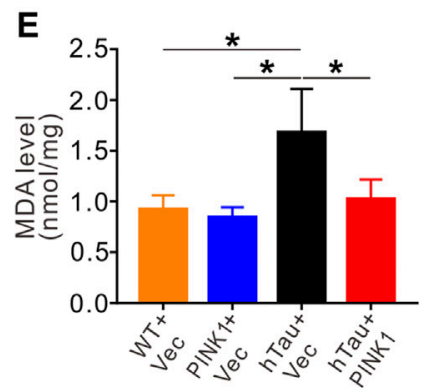

FIGURE 7 | PINK1 reduces tau accumulation in mitochondria and rescues mitochondrial disorders. (A-C) The levels of exogenous tau ( 106 kDa, human tau) $(\mathbf{A}, \mathbf{B})$ or Parkin $(\mathbf{A}, \mathbf{C})$ in both cytoplasm and mitochondria fraction of mice hippocampal CA1 region were detected by Western blot and quantitative analysis. Unpaired t-tests. ${ }^{\star} p<0.05,{ }^{\star \star} p<0.01$. (D, E) Overexpression of PINK1 reversed the decreased ATP levels (D) or increased MDA levels (E) in hTau mice. One-way ANOVA followed by Tukey multiple-comparisons tests. ${ }^{*} p<0.05$. All data were presented as mean \pm SD. $n=3$ mice for each group.

membrane protein 1 (LAMP1), lysosomal proteases cathepsin D, autophagy receptor OPTN and NDP52, thus leading to the clearance of $A \beta$ plaques (Du et al., 2017). SHSY5Y cells with PINK1 knockdown showed decreased mRNA levels of ATG5, ATG6, ATG7, LC3A, P62, LAMP1, and LAMP2 (Parganlija et al., 2014). PINK1 was also reported to directly interact with Beclin1 to promote autophagy (Michiorri et al., 2010). Taken together, our results indicate that PINK1 promotes degradation of tau via ALP. After pharmaceutical blockade of the fusion between autophagosome and lysosome by $\mathrm{CQ}$, we found that the decreased hTau proteins in hTau and PINK1overexpressed mice, including total and phosphorylated tau in whole tissue homogenate, soluble and insoluble portions, were all reversed. In contrast, inhibiting proteasome by MG132 just induced partial increase of hTau proteins in hTau and PINK1-overexpressed mice. This increase was mainly observed in the soluble portion, which is in accord with the idea that the proteasome has a limited ability to deal with oligomeric and aggregated proteins (Kirkin et al., 2009). CQ also reversed the PINK1induced improvements on cognitive impairment. Overall, the above findings verified that PINK1 relies on ALP to clear abnormal accumulated hTau proteins and ameliorate cognitive deficits. Thus, in future research, we will explore the detailed mechanism by which PINK1 overexpression leads to the degradation of tau via ALP.

Previous research has shown that cytosolic PINK1 fragments enhanced Parkin-mediated ubiquitination and degradation of Parkin substrates in neuroblastoma cells and human brain lysates (Xiong et al., 2009), and bioinformatic analysis presented the potential involvement of Parkin in the ubiquitination of tau (Kumar and Kumar, 2019). Researchers have also shown that Parkin brings about Lys63-linked polyubiquitination of misfolded proteins and leads to their clearance via the autophagy pathway (Olzmann and Chin, 2008; Khandelwal et al., 2011). In this study, a CoIP experiment revealed upregulated ubiquitination of tau protein in the hippocampal CA1 region of mice with PINK1 overexpression (Supplementary Figure S6).

Our previous studies found that hTau can accumulate in mitochondria, inhibit mitophagy, disrupt mitochondrial dynamics, and induce mitochondrial dysfunction in cellular or animal models overexpressing hTau (Hu et al., 2016; Li et al., 2016), which are considered drivers of synaptic dysfunction and cognitive decline in AD (John and Reddy, 2021; Sharma et al., 2021). In this study, we verified the pathological accumulation of tau in mitochondria, which was reported to induce mitochondrial dysfunction, thus contributing to synaptic impairment and memory deficits in 
mice (Torres et al., 2021). PINK1 has been widely reported to play vital roles in maintaining mitochondrial homeostasis (Voigt et al., 2016; Arena and Valente, 2017). Parkin is a cytosolic member of E3 ubiquitin ligase family, and overexpression PINK1 can recruit Parkin to mitochondria and activate it via phosphorylation of its UBL domain. Then, Parkin transfers ubiquitin chains to the mitochondrial outer membrane to induce the elimination of mitochondria through mitophagy (Okatsu et al., 2012; Du et al., 2017; Gundogdu et al., 2021). Our study showed that PINK1 reduced hTau accumulation in mitochondria. We observed mitochondrial dysfunction in hTau mice, which was rescued following overexpression of PINK1, possibly because of PINK1-induced reduction of intracellular tau accumulation (Li et al., 2016; Guha et al., 2020; Szabo et al., 2020) and the direct protective effects of PINK1 on mitochondria (Voigt et al., 2016; Arena and Valente, 2017).

It has been extensively reported that tau accumulation induces neuron loss and synaptic impairments, which are closely related to cognitive deficits in $\mathrm{AD}$ (Iqbal and Grundke-Iqbal, 2002; Giannakopoulos et al., 2003; Yin et al., 2016). We also found that overexpression of hTau or P301L hTau activated STAT1 and inactivated STAT3 to inhibit the expression of NMDARs, thus inducing dendritic plasticity deficits, including LTP suppression and spine density decrease, and memory deficits (Li et al., 2019; Hong et al., 2020; Wan et al., 2021). In this study, PINK1 overexpression rescued neuron loss and synaptic damage, and ameliorated cognitive impairments by promoting the degradation of accumulated tau in the autophagy pathway, reducing tau accumulation in mitochondria and alleviating mitochondrial disorders. These findings, together with the previous finding that PINK1 decreased $A \beta$ level in transgenic mAPP mice (Du et al., 2017), indicate the potential of PINK1 as a therapeutic target for $\mathrm{AD}$ treatment.

\section{REFERENCES}

Abdelfatah, S., Abdellatif, M., Abel, S., Ahmed, Z. M., Alves, S., Araki, Y., et al. (2021). Guidelines for the Use and Interpretation of Assays for Monitoring Autophagy (4th Edition)1. Autophagy 17 (1), 1-382. doi:10.1080/ 15548627.2020.1797280

Akabane, S., Matsuzaki, K., Yamashita, S.-I., Arai, K., Okatsu, K., Kanki, T., et al. (2016). Constitutive Activation of PINK1 Protein Leads to ProteasomeMediated and Non-Apoptotic Cell Death Independently of Mitochondrial Autophagy. J. Biol. Chem. 291 (31), 16162-16174. doi:10.1074/ jbc.M116.714923

Andorfer, C., Kress, Y., Espinoza, M., De Silva, R., Tucker, K. L., Barde, Y.-A., et al. (2003). Hyperphosphorylation and Aggregation of Tau in Mice Expressing Normal Human Tau Isoforms. J. Neurochem. 86 (3), 582-590. doi:10.1046/ j.1471-4159.2003.01879.x

Arena, G., and Valente, E. M. (2017). PINK1 in the Limelight: Multiple Functions of an Eclectic Protein in Human Health and Disease. J. Pathol. 241 (2), 251-263. doi:10.1002/path.4815

Baek, M., Choe, Y.-J., Bannwarth, S., Kim, J., Maitra, S., Dorn, G. W., et al. (2021). TDP-43 and PINK1 Mediate CHCHD10S59L Mutation-Induced Defects in Drosophila and In Vitro. Nat. Commun. 12 (1), 1924. doi:10.1038/s41467-02122145-9

Bejanin, A., Schonhaut, D. R., La Joie, R., Kramer, J. H., Baker, S. L., Sosa, N., et al. (2017). Tau Pathology and Neurodegeneration Contribute to Cognitive

\section{DATA AVAILABILITY STATEMENT}

The original contributions presented in the study are included in the article/Supplementary Material, Further inquiries can be directed to the corresponding authors.

\section{ETHICS STATEMENT}

The animal study was reviewed and approved by the Ethics Committee of Tongji Medical College, Huazhong University of Science and Technology.

\section{AUTHOR CONTRIBUTIONS}

GL and GPL proposed and designed the research project, and wrote the manuscript. XJ and YW performed most of the experiments, and conducted data analysis and graphics production. RM, YC, LL, and JZ contributed to the experiments. All authors contributed to the article and approved the submitted version.

\section{FUNDING}

This work is supported by the grant from the National Natural Science Foundation of China (No. 81974161).

\section{SUPPLEMENTARY MATERIAL}

The Supplementary Material for this article can be found online at: https://www.frontiersin.org/articles/10.3389/fcell.2021.736267/ full\#supplementary-material

Impairment in Alzheimer's Disease. Brain 140 (12), 3286-3300. doi:10.1093/ brain/awx 243

Campos, J. C., Baehr, L. M., Ferreira, N. D., Bozi, L. H. M., Andres, A. M., Ribeiro, M. A. C., et al. (2020). $\beta 2$-Adrenoceptor Activation Improves Skeletal Muscle Autophagy in Neurogenic Myopathy. FASEB j. 34 (4), 5628-5641. doi:10.1096/ fj.201902305R

Chen, Y., Chen, Y., Zhang, J., Cao, P., Su, W., Deng, Y., et al. (2020). Fusobacterium Nucleatum Promotes Metastasis in Colorectal Cancer by Activating Autophagy Signaling via the Upregulation of CARD3 Expression. Theranostics 10 (1), 323-339. doi:10.7150/thno.38870

Cheng, J., North, B. J., Zhang, T., Dai, X., Tao, K., Guo, J., et al. (2018). The Emerging Roles of Protein Homeostasis-Governing Pathways in Alzheimer's Disease. Aging Cell 17 (5), e12801. doi:10.1111/acel.12801

Chidambaram, S. B., Rathipriya, A. G., Bolla, S. R., Bhat, A., Ray, B., Mahalakshmi, A. M., et al. (2019). Dendritic Spines: Revisiting the Physiological Role. Prog. Neuro-Psychopharmacology Biol. Psychiatry 92, 161-193. doi:10.1016/ j.pnpbp.2019.01.005

Choi, J., Ravipati, A., Nimmagadda, V., Schubert, M., Castellani, R. J., and Russell, J. W. (2014). Potential Roles of PINK1 for Increased PGC-1 $\alpha$ Mediated Mitochondrial Fatty Acid Oxidation and Their Associations with Alzheimer Disease and Diabetes. Mitochondrion 18, 41-48. doi:10.1016/j.mito.2014.09.005

Congdon, E. E., and Sigurdsson, E. M. (2018). Tau-Targeting Therapies for Alzheimer Disease. Nat. Rev. Neurol. 14 (7), 399-415. doi:10.1038/s41582018-0013-z 
Du, F., Yu, Q., Yan, S., Hu, G., Lue, L.-F., Walker, D. G., et al. (2017). PINK1 Signalling Rescues Amyloid Pathology and Mitochondrial Dysfunction in Alzheimer's Disease. Brain 140 (12), 3233-3251. doi:10.1093/brain/awx258

Fagerberg, L., Hallström, B. M., Oksvold, P., Kampf, C., Djureinovic, D., Odeberg, J., et al. (2014). Analysis of the Human Tissue-Specific Expression by GenomeWide Integration of Transcriptomics and Antibody-Based Proteomics. Mol. Cell Proteomics 13 (2), 397-406. doi:10.1074/mcp.M113.035600

Fang, E. F., Hou, Y., Palikaras, K., Adriaanse, B. A., Kerr, J. S., Yang, B., et al. (2019). Mitophagy Inhibits Amyloid- $\beta$ and Tau Pathology and Reverses Cognitive Deficits in Models of Alzheimer's Disease. Nat. Neurosci. 22 (3), 401-412. doi:10.1038/s41593-018-0332-9

Ferrer, I., Andrés-Benito, P., Zelaya, M. V., Aguirre, M. E. E., Carmona, M., Ausín, K., et al. (2020). Familial Globular Glial Tauopathy Linked to MAPT Mutations: Molecular Neuropathology and Seeding Capacity of a Prototypical Mixed Neuronal and Glial Tauopathy. Acta Neuropathol. 139 (4), 735-771. doi:10.1007/s00401-019-02122-9

Giannakopoulos, P., Herrmann, F. R., Bussiere, T., Bouras, C., Kovari, E., Perl, D. P., et al. (2003). Tangle and Neuron Numbers, but Not Amyloid Load, Predict Cognitive Status in Alzheimer's Disease. Neurology 60 (9), 1495-1500. doi:10.1212/01.WNL.0000063311.58879.01

Goedert, M., Spillantini, M. G., Cairns, N. J., and Crowther, R. A. (1992). Tau Proteins of Alzheimer Paired Helical Filaments: Abnormal Phosphorylation of All Six Brain Isoforms. Neuron 8 (1), 159-168. doi:10.1016/0896-6273(92)90117-v

Götz, J., Halliday, G., and Nisbet, R. M. (2019). Molecular Pathogenesis of the Tauopathies. Annu. Rev. Pathol. Mech. Dis. 14 (1), 239-261. doi:10.1146/ annurev-pathmechdis-012418-012936

Grassi, D., Diaz-Perez, N., Volpicelli-Daley, L. A., and Lasmézas, C. I. (2019). Pasyn $^{*}$ Mitotoxicity Is Linked to MAPK Activation and Involves Tau Phosphorylation and Aggregation at the Mitochondria. Neurobiol. Dis. 124, 248-262. doi:10.1016/j.nbd.2018.11.015

Guha, S., Johnson, G. V. W., and Nehrke, K. (2020). The Crosstalk between Pathological Tau Phosphorylation and Mitochondrial Dysfunction as a Key to Understanding and Treating Alzheimer's Disease. Mol. Neurobiol. 57 (12), 5103-5120. doi:10.1007/s12035-020-02084-0

Gundogdu, M., Tadayon, R., Salzano, G., Shaw, G. S., and Walden, H. (2021). A Mechanistic Review of Parkin Activation. Biochim. Biophys. Acta (Bba) - Gen. Subjects 1865 (6), 129894. doi:10.1016/j.bbagen.2021.129894

Guo, T., Zhang, D., Zeng, Y., Huang, T. Y., Xu, H., and Zhao, Y. (2020). Molecular and Cellular Mechanisms Underlying the Pathogenesis of Alzheimer's Disease. Mol. Neurodegeneration 15 (1), 40. doi:10.1186/s13024-020-00391-7

Han, Y., Wang, N., Kang, J., and Fang, Y. (2020). $\beta$-Asarone Improves Learning and Memory in A $\beta 1$-42-Induced Alzheimer's Disease Rats by Regulating PINK1-Parkin-Mediated Mitophagy. Metab. Brain Dis. 35 (7), 1109-1117. doi:10.1007/s11011-020-00587-2

Hong, X.-Y., Wan, H.-L., Li, T., Zhang, B.-G., Li, X.-G., Wang, X., et al. (2020). STAT3 Ameliorates Cognitive Deficits by Positively Regulating the Expression of NMDARs in a Mouse Model of FTDP-17. Sig Transduct Target. Ther. 5 (1), 213-295. doi:10.1038/s41392-020-00290-9

Hu, Y., Li, X.-C., Wang, Z.-H., Luo, Y., Zhang, X., Liu, X.-P., et al. (2016). Tau Accumulation Impairs Mitophagy via Increasing Mitochondrial Membrane Potential and Reducing Mitochondrial Parkin. Oncotarget 7 (14), 17356-17368. doi:10.18632/oncotarget.7861

Iqbal, K., and Grundke-Iqbal, I. (2002). Neurofibrillary Pathology Leads to Synaptic Loss and Not the Other Way Around in Alzheimer Disease. J. Alzheimer's Dis. 4 (3), 235-238. doi:10.3233/JAD-2002-4313

John, A., and Reddy, P. H. (2021). Synaptic Basis of Alzheimer's Disease: Focus on Synaptic Amyloid Beta, P-Tau and Mitochondria. Ageing Res. Rev. 65, 101208. doi:10.1016/j.arr.2020.101208

Khalil, B., El Fissi, N., Aouane, A., Cabirol-Pol, M.-J., Rival, T., and Liévens, J.-C. (2015). PINK1-Induced Mitophagy Promotes Neuroprotection in Huntington's Disease. Cell Death Dis 6 (1), e1617. doi:10.1038/cddis.2014.581

Khandelwal, P. J., Herman, A. M., Hoe, H.-S., Rebeck, G. W., and Moussa, C. E. H. (2011). Parkin Mediates Beclin-Dependent Autophagic Clearance of Defective Mitochondria and Ubiquitinated A in AD Models. Hum. Mol. Genet. 20 (11), 2091-2102. doi:10.1093/hmg/ddr091

Kirkin, V., McEwan, D. G., Novak, I., and Dikic, I. (2009). A Role for Ubiquitin in Selective Autophagy. Mol. Cel 34 (3), 259-269. doi:10.1016/ j.molcel.2009.04.026
Korhonen, P., van Groen, T., Thornell, A., Kyrylenko, S., Soininen, M.-L., Ojala, J., et al. (2011). Characterization of a Novel Transgenic Rat Carrying Human Tau with Mutation P301L. Neurobiol. Aging 32 (12), 2314-2315. doi:10.1016/ j.neurobiolaging.2009.12.022

Kumar, D., and Kumar, P. (2019). A $\beta$, Tau, and $\alpha$-Synuclein Aggregation and Integrated Role of PARK2 in the Regulation and Clearance of Toxic Peptides. Neuropeptides 78, 101971. doi:10.1016/j.npep.2019.101971

Lasagna-Reeves, C. A., Castillo-Carranza, D. L., Sengupta, U., Clos, A. L., Jackson, G. R., and Kayed, R. (2011). Tau Oligomers Impair Memory and Induce Synaptic and Mitochondrial Dysfunction in Wild-type Mice. Mol. Neurodegeneration 6, 39. doi:10.1186/1750-1326-6-39

Li, X.-C., Hu, Y., Wang, Z.-H., Luo, Y., Zhang, Y., Liu, X.-P., et al. (2016). Human Wild-type Full-Length Tau Accumulation Disrupts Mitochondrial Dynamics and the Functions via Increasing Mitofusins. Sci. Rep. 6 (1), 24756. doi:10.1038/ srep24756

Li, X. G., Hong, X. Y., Wang, Y. L., Zhang, S. J., Zhang, J. F., Li, X. C., et al. (2019). Tau Accumulation Triggers STAT 1-Dependent Memory Deficits by Suppressing NMDA Receptor Expression. EMBO Rep. 20 (6), e47202. doi:10.15252/embr.201847202

Li, X, Chen, C., Zhan, X., Li, B., Zhang, Z., Li, S., et al. (2021). R13 Preserves Motor Performance in SOD1(G93A) Mice by Improving Mitochondrial Function. Theranostics 11 (15), 7294-7307. doi:10.7150/thno.56070

Liang, C., Mu, Y., Tian, H., Wang, D., Zhang, S., Wang, H., et al. (2021). MicroRNA-140 Silencing Represses the Incidence of Alzheimer's Disease. Neurosci. Lett. 758, 135674. doi:10.1016/j.neulet.2021.135674

Lu, H., Liufu, N., Dong, Y., Xu, G., Zhang, Y., Shu, L., et al. (2017). Sevoflurane Acts on Ubiquitination-Proteasome Pathway to Reduce Postsynaptic Density 95 Protein Levels in Young Mice. Anesthesiology 127 (6), 961-975. doi:10.1097/ ALN.0000000000001889

Manczak, M., Kandimalla, R., Yin, X., and Reddy, P. H. (2018). Hippocampal Mutant APP and Amyloid Beta-Induced Cognitive Decline, Dendritic Spine Loss, Defective Autophagy, Mitophagy and Mitochondrial Abnormalities in a Mouse Model of Alzheimer's Disease. Hum. Mol. Genet. 27 (8), 1332-1342. doi:10.1093/hmg/ddy042

McLelland, G.-L., Soubannier, V., Chen, C. X., McBride, H. M., and Fon, E. A. (2014). Parkin and PINK1 Function in a Vesicular Trafficking Pathway Regulating Mitochondrial Quality Control. EMBO J. 33 (4), 282-295. doi: $10.1002 / \mathrm{embj} .201385902$

McWilliams, T. G., Prescott, A. R., Montava-Garriga, L., Ball, G., Singh, F., Barini, E., et al. (2018). Basal Mitophagy Occurs Independently of PINK1 in Mouse Tissues of High Metabolic Demand. Cel Metab. 27 (2), 439-449. doi:10.1016/ j.cmet.2017.12.008

Michiorri, S., Gelmetti, V., Giarda, E., Lombardi, F., Romano, F., Marongiu, R., et al. (2010). The Parkinson-Associated Protein PINK1 Interacts with Beclin1 and Promotes Autophagy. Cell Death Differ 17 (6), 962-974. doi:10.1038/ cdd.2009.200

Mise, A., Yoshino, Y., Yamazaki, K., Ozaki, Y., Sao, T., Yoshida, T., et al. (2017). TOMM40 and APOE Gene Expression and Cognitive Decline in Japanese Alzheimer's Disease Subjects. J. Alzheimer's Dis. 60 (3), 1107-1117. doi:10.3233/JAD-170361

Morris, R. (1984). Developments of a Water-Maze Procedure for Studying Spatial Learning in the Rat. J. Neurosci. Methods 11 (1), 47-60. doi:10.1016/01650270(84)90007-4

Ochi, S., Iga, J.-I., Funahashi, Y., Yoshino, Y., Yamazaki, K., Kumon, H., et al. (2020). Identifying Blood Transcriptome Biomarkers of Alzheimer's Disease Using Transgenic Mice. Mol. Neurobiol. 57 (12), 4941-4951. doi:10.1007/ s12035-020-02058-2

Okatsu, K., Oka, T., Iguchi, M., Imamura, K., Kosako, H., Tani, N., et al. (2012). PINK1 Autophosphorylation upon Membrane Potential Dissipation Is Essential for Parkin Recruitment to Damaged Mitochondria. Nat. Commun. 3 (1), 1016. doi:10.1038/ncomms2016

Olzmann, J. A., and Chin, L.-S. (2008). Parkin-mediated K63-Linked Polyubiquitination: A Signal for Targeting Misfolded Proteins to the Aggresome-Autophagy Pathway. Autophagy 4 (1), 85-87. doi:10.4161/auto.5172

Pakpian, N., Phopin, K., Kitidee, K., Govitrapong, P., and Wongchitrat, P. (2020). Alterations in Mitochondrial Dynamic-Related Genes in the Peripheral Blood of Alzheimer's Disease Patients. Curr. Alzheimer Res. 17 (7), 616-625. doi:10.2174/1567205017666201006162538 
Parganlija, D., Klinkenberg, M., Domínguez-Bautista, J., Hetzel, M., Gispert, S., Chimi, M. A., et al. (2014). Loss of PINK1 Impairs Stress-Induced Autophagy and Cell Survival. PLoS One 9 (4), e95288. doi:10.1371/journal.pone.0095288

Quinn, P. M. J., Moreira, P. I., Ambrósio, A. F., and Alves, C. H. (2020). PINK1/ PARKIN Signalling in Neurodegeneration and Neuroinflammation. Acta Neuropathol. Commun. 8 (1), 189. doi:10.1186/s40478-020-01062-w

Rasool, S., and Trempe, J.-F. (2018). New Insights into the Structure of PINK1 and the Mechanism of Ubiquitin Phosphorylation. Crit. Rev. Biochem. Mol. Biol. 53 (5), 515-534. doi:10.1080/10409238.2018.1491525

Reddy, P. H., Yin, X., Manczak, M., Kumar, S., Pradeepkiran, J. A., Vijayan, M., et al. (2018). Mutant APP and Amyloid Beta-Induced Defective Autophagy, Mitophagy, Mitochondrial Structural and Functional Changes and Synaptic Damage in Hippocampal Neurons from Alzheimer's Disease. Hum. Mol. Genet. 27 (14), 2502-2516. doi:10.1093/hmg/ddy154

Schlegel, K., Awwad, K., Heym, R. G., Holzinger, D., Doell, A., Barghorn, S., et al. (2019). N368-Tau Fragments Generated by Legumain Are Detected Only in Trace Amount in the Insoluble Tau Aggregates Isolated from AD Brain. Acta Neuropathol. Commun. 7 (1), 177. doi:10.1186/s40478-019-0831-2

Sharma, C., Kim, S., Nam, Y., Jung, U. J., and Kim, S. R. (2021). Mitochondrial Dysfunction as a Driver of Cognitive Impairment in Alzheimer's Disease. Int. J. Mol. Sci. 22 (9), 4850. doi:10.3390/ijms22094850

Simic, G., Kostovic, I., Winblad, B., and Bogdanovic, N. (1997). Volume and Number of Neurons of the Human Hippocampal Formation in Normal Aging and Alzheimer's Disease. J. Comp. Neurol. 379 (4), 482-494. doi:10.1002/(sici) 1096-9861(19970324)379:4<482::aid-cne2>3.0.co;2-z

Sliter, D. A., Martinez, J., Hao, L., Chen, X., Sun, N., Fischer, T. D., et al. (2018). Parkin and PINK1 Mitigate STING-Induced Inflammation. Nature 561 (7722), 258-262. doi:10.1038/s41586-018-0448-9

Szabo, L., Eckert, A., and Grimm, A. (2020). Insights into Disease-Associated Tau Impact on Mitochondria. Int. J. Mol. Sci. 21 (17), 6344. doi:10.3390/ ijms21176344

Tapia-Rojas, C., Cabezas-Opazo, F., Deaton, C. A., Vergara, E. H., Johnson, G. V. W., and Quintanilla, R. A. (2019). It's All about Tau. Prog. Neurobiol. 175, 54-76. doi:10.1016/j.pneurobio.2018.12.005

Torres, A. K., Jara, C., Olesen, M. A., and Tapia-Rojas, C. (2021). Pathologically Phosphorylated Tau at S396/404 (PHF-1) Is Accumulated inside of Hippocampal Synaptic Mitochondria of Aged Wild-Type Mice. Sci. Rep. 11 (1), 4448. doi:10.1038/s41598-021-83910-w

Valente, E. M., Abou-Sleiman, P. M., Caputo, V., Muqit, M. M. K., Harvey, K., Gispert, S., et al. (2004). Hereditary Early-Onset Parkinson's Disease Caused by Mutations in PINK1. Science 304 (5674), 1158-1160. doi:10.1126/ science.1096284

Voigt, A., Berlemann, L. A., and Winklhofer, K. F. (2016). The Mitochondrial Kinase PINK1: Functions beyond Mitophagy. J. Neurochem. 139 (Suppl. 1), 232-239. doi:10.1111/jnc.13655

Wan, H.-L., Hong, X.-Y., Zhao, Z.-H., Li, T., Zhang, B.-G., Liu, Q., et al. (2021). STAT3 Ameliorates Cognitive Deficits via Regulation of NMDAR Expression in an Alzheimer's Disease Animal Model. Theranostics 11 (11), 5511-5524. doi:10.7150/thno.56541

West, M. J., Coleman, P. D., Flood, D. G., and Troncoso, J. C. (1994). Differences in the Pattern of Hippocampal Neuronal Loss in normal Ageing and Alzheimer's Disease. The Lancet 344 (8925), 769-772. doi:10.1016/S0140-6736(94)92338-8

Xiong, H., Wang, D., Chen, L., Choo, Y. S., Ma, H., Tang, C., et al. (2009). Parkin, PINK1, and DJ-1 Form a Ubiquitin E3 Ligase Complex Promoting Unfolded Protein Degradation. J. Clin. Invest. 119 (3), 650-660. doi:10.1172/JCI37617

Ye, M., Zhou, D., Zhou, Y., and Sun, C. (2015). Parkinson's Disease-Associated PINK1G309D Mutation Increases Abnormal Phosphorylation of Tau. Iubmb Life 67 (4), 286-290. doi:10.1002/iub.1367

Yin, Y., Gao, D., Wang, Y., Wang, Z.-H., Wang, X., Ye, J., et al. (2016). Tau Accumulation Induces Synaptic Impairment and Memory Deficit by Calcineurin-Mediated Inactivation of Nuclear CaMKIV/CREB Signaling. Proc. Natl. Acad. Sci. USA 113 (26), E3773-E3781. doi:10.1073/ pnas. 1604519113

Zhang, Y., Gao, J., Chung, K. K. K., Huang, H., Dawson, V. L., and Dawson, T. M. (2000). Parkin Functions as an E2-dependent Ubiquitin- Protein Ligase and Promotes the Degradation of the Synaptic Vesicle-Associated Protein, CDCrel1. Proc. Natl. Acad. Sci. 97 (24), 13354-13359. doi:10.1073/pnas.240347797

Zhao, N., Yan, Q.-W., Xia, J., Zhang, X.-L., Li, B.-X., Yin, L.-Y., et al. (2020). Treadmill Exercise Attenuates A $\beta$-Induced Mitochondrial Dysfunction and Enhances Mitophagy Activity in APP/PS1 Transgenic Mice. Neurochem. Res. 45 (5), 1202-1214. doi:10.1007/s11064-020-03003-4

Zheng, J., Akbari, M., Schirmer, C., Reynaert, M.-L., Loyens, A., Lefebvre, B., et al. (2020). Hippocampal Tau Oligomerization Early in Tau Pathology Coincides with a Transient Alteration of Mitochondrial Homeostasis and DNA Repair in a Mouse Model of Tauopathy. Acta Neuropathol. Commun. 8 (1), 25. doi:10.1186/s40478-020-00896-8

Conflict of Interest: The authors declare that the research was conducted in the absence of any commercial or financial relationships that could be construed as a potential conflict of interest.

Publisher's Note: All claims expressed in this article are solely those of the authors and do not necessarily represent those of their affiliated organizations, or those of the publisher, the editors, and the reviewers. Any product that may be evaluated in this article, or claim that may be made by its manufacturer, is not guaranteed or endorsed by the publisher.

Copyright (C) 2022 Jiang, Wu, Ma, Chang, Li, Zhu, Liu and Li. This is an open-access article distributed under the terms of the Creative Commons Attribution License (CC $B Y$ ). The use, distribution or reproduction in other forums is permitted, provided the original author(s) and the copyright owner(s) are credited and that the original publication in this journal is cited, in accordance with accepted academic practice. No use, distribution or reproduction is permitted which does not comply with these terms. 